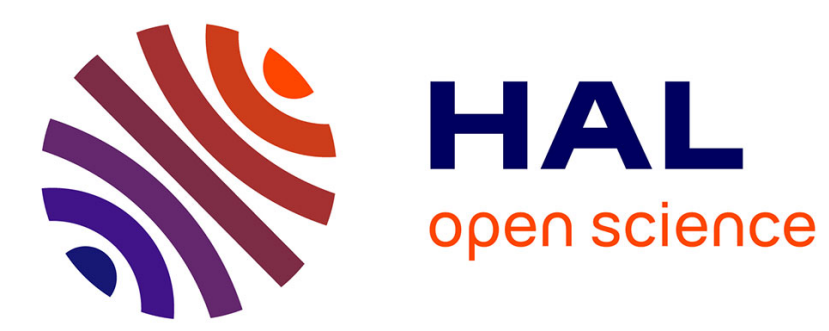

\title{
A new class of probability distributions via cosine and sine functions with applications
}

Christophe Chesneau, Hassan S Bakouch, Tassaddaq Hussain

\section{To cite this version:}

Christophe Chesneau, Hassan S Bakouch, Tassaddaq Hussain. A new class of probability distributions via cosine and sine functions with applications. 2017. hal-01569293

\section{HAL Id: hal-01569293 \\ https://hal.science/hal-01569293}

Preprint submitted on 26 Jul 2017

HAL is a multi-disciplinary open access archive for the deposit and dissemination of scientific research documents, whether they are published or not. The documents may come from teaching and research institutions in France or abroad, or from public or private research centers.
L'archive ouverte pluridisciplinaire HAL, est destinée au dépôt et à la diffusion de documents scientifiques de niveau recherche, publiés ou non, émanant des établissements d'enseignement et de recherche français ou étrangers, des laboratoires publics ou privés. 


\title{
A new class of probability distributions via cosine and sine functions with applications
}

\author{
Christophe Chesneau ${ }^{\mathrm{a}}$, Hassan S. Bakouch ${ }^{\mathrm{b}, *}$, Tassaddaq Hussain ${ }^{\mathrm{c}}$ \\ ${ }^{a} L M N O$, University of Caen, France \\ ${ }^{b}$ Department of Mathematics, Faculty of Science, Tanta University, Tanta, Egypt \\ ${ }^{c}$ Mirpur University of Science and Technology (MUST) Mirpur, 10250 (AJK), Pakistan
}

\begin{abstract}
In this paper, we introduce a new class of (probability) distributions, based on a cosine-sine transformation, obtained by compounding a baseline distribution with cosine and sine functions. Some of its properties are explored. A special focus is given to a particular cosine-sine transformation using the exponential distribution as baseline. Estimations of parameters of a particular cosine-sine exponential distribution are performed via the maximum likelihood estimation method. A complete simulation study investigates the performances of these estimates. Applications are given for four real data sets, showing a better fit in comparison to some existing distributions based on some of goodness-of-fit tests.
\end{abstract}

Keywords: Cosine-sine transformation, Estimation, Goodness-of-fit statistics, Hazard rate function, Statistical distributions.

2000 MSC: 60E05, 62E15, 62F10

\section{Introduction}

The statistical literature contains a plethora of (probability) distributions for modeling different real life random phenomenon in several areas such as engineering, actuarial, medical sciences, demography, economics, finance and insurance. Since no particular distribution is appropriate for modeling every phenomenon, the list of new distributions with a high degree of flexibility is growing every year. Some of the recent univariate continuous distributions can be found in $[6,7],[8]$ and $[20]$. As a matter of fact, the statistical literature has a lack of distributions which are based on trigonometric functions; most of them are based on algebraic functions. Among the distributions using trigonometric distributions, let us mention the von Mises distribution (see [3, "von Mises Distribution" Ch. 41, 189-191]), the distributions introduced by [4] to analyze circular data, the beta-type distributions using some trigonometric functions proposed by [17], the circular Cauchy distribution introduced by [9] and the sine square distribution explored by [1]. However, the increased interest of data analysis, and directional data analysis in particular, motivates the development of new approaches. The one explored by [14] provides a modem alternative. They introduced a new class of distributions obtained by compounding a baseline distribution with the sine function called the SS transformation. For any cumulative distribution function (cdf) $F(x)$, it is defined by $G(x)=\sin \left(\frac{\pi}{2} F(x)\right)$. Taking $F(x)$ as the cdf of an exponential distribution, [14] propose a new distribution to solve a parametric estimation problem inherent to bladder cancer patients data, and it is shown that it has a better fit compared to several well-known distributions.

Based on the motivations above, we propose a generalization of the SS transformation, using both cosine and sine functions, which can give many of new trigonometric distributions, as shall be pointed out. Several tuning parameters increasing the degree of flexibility of the original SS transformation.

\footnotetext{
${ }^{\text {th }}$ Corresponding author

Email addresses: christophe.chesneau@unicaen.fr (Christophe Chesneau), hnbakouch@yahoo.com (Hassan S. Bakouch), taskho2000@yahoo.com (Tassaddaq Hussain)
} 
We exhibit the associated probability density function (pdf) and the hazard rate function (hrf). A decomposition of the cdf is proposed. By considering two different sets of parameters, we introduce two particular CS transformations, called the CS1 and CS2 transformations. New distributions are derived by considering well-known distributions (uniform, exponential, normal, arcsine...). Some of their mathematical properties are studied. A special focus is given when the baseline distribution is the exponential distribution. For this case, we discuss a parametric estimation via the maximum likelihood estimation method. A simulation study is performed to evaluate the performances of these estimates using different sample sizes and two criteria: the average bias the average mean square error. Then we show the applicability of a specific cosine-sine exponential distribution by considering four data sets. They are fitted to our distribution and other recent distributions based on some wellknown goodness-of-fit statistics $\left(A_{0}^{*}, W_{0}^{*}\right.$, AIC, AICc, HQIC, CAIC). Moreover, it demonstrates a better flexibility, which is illustrated among the compared distributions.

The rest of the paper is organized as follows: Section 2 presents the general form of our cosine-sine transformation. New distributions are derived in Sections 3 and 4 using particular parametrization. In Section 5, the performance of the estimates of the parameters of two types of cosine-sine exponential distributions is investigated using a simulation study. In Section 6 , four real data sets are fitted to a specific cosine-sine exponential distribution, with comparison to other recent distributions based on some of goodness-of-fit statistics. The proofs of our main results are given in Appendix.

\section{CS transformation}

In the present study, using a cdf $F(x)$, we introduce the following cosine-sine transformation :

$$
G(x)=\frac{(\alpha+\gamma) \sin \left(\frac{\pi}{2} F(x)\right)}{\alpha+\beta \cos \left(\frac{\pi}{2} F(x)\right)+\gamma \sin \left(\frac{\pi}{2} F(x)\right)+\theta \cos \left(\frac{\pi}{2} F(x)\right) \sin \left(\frac{\pi}{2} F(x)\right)} .
$$

Remark that the last term in the denominator can be expressed as : $\cos \left(\frac{\pi}{2} F(x)\right) \sin \left(\frac{\pi}{2} F(x)\right)=$ $\frac{1}{2} \sin (\pi F(x))$. We will call the transformation (1) as the CS transformation for frequently used purposed.

Theorem 1. If $\alpha \geq 0, \beta \geq 0, \gamma \geq 0, \theta \geq 0, \alpha+\gamma>0$ and $\alpha+\beta>0$, the function $G(x)$ given by (1) possesses the properties of a cdf.

The proof of Theorem 1 is given in Appendix.

Note that, taking $\alpha>0, \beta=0, \gamma=0$ and $\theta=0, G(x)$ becomes the SS transformation of [14]. Further specific choices of parameters, yielding new cdfs, will be studied later. Observe that $G(x) \sim \frac{\pi(\alpha+\gamma)}{2(\alpha+\beta)} F(x)$, when $F(x) \rightarrow 0$, showing a first look to the flexibility of the CS distribution.

The following results follow from Theorem 1 . Let $f(x)$ be a pdf associated to $F(x)$.

- The pdf associated to $G(x)$ is given by

$$
g(x)=\frac{\pi(\alpha+\gamma)\left(\beta+\alpha \cos \left(\frac{\pi}{2} F(x)\right)+\theta\left(\sin \left(\frac{\pi}{2} F(x)\right)\right)^{3}\right) f(x)}{2\left(\alpha+\beta \cos \left(\frac{\pi}{2} F(x)\right)+\gamma \sin \left(\frac{\pi}{2} F(x)\right)+\theta \cos \left(\frac{\pi}{2} F(x)\right) \sin \left(\frac{\pi}{2} F(x)\right)\right)^{2}} .
$$

Hence $g(x)$ is a weighted pdf of the form $g(x)=c w(x) f(x)$, where $w(x)$ is the weight

$$
\begin{aligned}
& w(x)=\frac{\beta+\alpha \cos \left(\frac{\pi}{2} F(x)\right)+\theta\left(\sin \left(\frac{\pi}{2} F(x)\right)\right)^{3}}{\left(\alpha+\beta \cos \left(\frac{\pi}{2} F(x)\right)+\gamma \sin \left(\frac{\pi}{2} F(x)\right)+\theta \cos \left(\frac{\pi}{2} F(x)\right) \sin \left(\frac{\pi}{2} F(x)\right)\right)^{2}} \text { and } c \text { the normalization constant: } \\
& c=\frac{\pi(\alpha+\gamma)}{2} .
\end{aligned}
$$

- The hrf associated to $G(x)$ is given by

$$
h(x)=\frac{\pi(\alpha+\gamma)\left(\beta+\alpha \cos \left(\frac{\pi}{2} F(x)\right)+\theta\left(\sin \left(\frac{\pi}{2} F(x)\right)\right)^{3}\right) f(x)}{2 q(x)},
$$


where

$$
\begin{aligned}
q(x) & =\left(\alpha+\beta \cos \left(\frac{\pi}{2} F(x)\right)+\gamma \sin \left(\frac{\pi}{2} F(x)\right)+\theta \cos \left(\frac{\pi}{2} F(x)\right) \sin \left(\frac{\pi}{2} F(x)\right)\right) \\
& \times\left(\alpha+\beta \cos \left(\frac{\pi}{2} F(x)\right)-\alpha \sin \left(\frac{\pi}{2} F(x)\right)+\theta \cos \left(\frac{\pi}{2} F(x)\right) \sin \left(\frac{\pi}{2} F(x)\right)\right) .
\end{aligned}
$$

From (1) and existing transformations, one can construct a wide variety of distributions. For instance, from a cdf $H(x)$, one can use the cdf $F(x)$ into (1) using

- the dilation transformation : $F(x)=H(\alpha x), \alpha>0$,

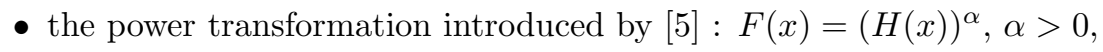

- the DUS transformation introduced by [13] : $F(x)=\frac{1}{e-1}\left(e^{H(x)}-1\right)$,

- the quadratic rank transmutation map (QRTM) introduced by [19], yielding the transformation : $F(x)=(1+\lambda) H(x)-\lambda(H(x))^{2}, \lambda \in[-1,1]$.

All these possibilities give new families of distributions using cosine and sine functions, with a potential interest in probability and statistics.

Proposition 1 below shows that the CS transformation can be expand as a non trivial sum of powers of the function $\sin \left(\frac{\pi}{2} F(x)\right)$.

Proposition 1. Let us set $\nu=\alpha+\beta+\gamma+\theta$. We have the following expansion:

$$
G(x)=\frac{\alpha+\gamma}{\nu} \sum_{k=0}^{+\infty} \sum_{\ell=0}^{k} \sum_{m=0}^{\ell} \sum_{q=0}^{m} \sum_{r=0}^{\ell-m} \sum_{s=0}^{k-\ell} \sum_{t=0}^{+\infty} a_{k, \ell, m, q, r, s, t}\left(\sin \left(\frac{\pi}{2} F(x)\right)\right)^{q+r+2 t+1},
$$

with

$$
a_{k, \ell, m, q, r, s, t}=\left(\begin{array}{c}
k \\
\ell
\end{array}\right)\left(\begin{array}{c}
\ell \\
m
\end{array}\right)\left(\begin{array}{c}
m \\
q
\end{array}\right)\left(\begin{array}{c}
\ell-m \\
r
\end{array}\right)\left(\begin{array}{c}
k-\ell \\
s
\end{array}\right)\left(\begin{array}{c}
(q+s) / 2 \\
t
\end{array}\right)\left(\frac{\beta}{\nu}\right)^{k-\ell}\left(\frac{\theta}{\nu}\right)^{m}\left(\frac{\gamma}{\nu}\right)^{\ell-m}(-1)^{q+r+s+t} .
$$

In the rest of the study, we focus our attention on two special cases of our CS transformation, both using a particular parametrization on the parameters $(\alpha, \beta, \gamma, \theta)$.

\section{The CS1 transformation}

An interesting special case of our CS transformation is with the parametrization $\alpha>0, \beta=0$, $\gamma=0$ and $\theta \geq 0$. Hence the cdf $G(x)$ (1) only depends on the parameters $\alpha$ and $\theta$. Using trigonometric formulas, we have

$$
G(x)=\frac{\alpha \sin \left(\frac{\pi}{2} F(x)\right)}{\alpha+\theta \cos \left(\frac{\pi}{2} F(x)\right) \sin \left(\frac{\pi}{2} F(x)\right)}=\frac{2 \alpha \sin \left(\frac{\pi}{2} F(x)\right)}{2 \alpha+\theta \sin (\pi F(x))} .
$$

An alternative expression of $G(x)$ is given by using the survival function $S(x)$ defined by $S(x)=$ $1-F(x)$ and standard trigonometric formulas:

$$
G(x)=\frac{2 \alpha \sin \left(\frac{\pi}{2}(1-S(x))\right)}{2 \alpha+\theta \sin (\pi(1-S(x)))}=\frac{2 \alpha \cos \left(\frac{\pi}{2} S(x)\right)}{2 \alpha+\theta \sin (\pi S(x))} .
$$

Again, note that, taking $\theta=0$, we obtain the SS transformation of [14]. We thus have more flexibility in our transformation thanks to the additional parameter $\theta$, opening new perspective in parametric estimation for instance. We will call the transformation (2) as the CS1 transformation for frequently used purposed. Using again trigonometric formulas, the associated pdf is

$$
g(x)=\frac{2 \pi \alpha\left(\alpha \cos \left(\frac{\pi}{2} F(x)\right)+\theta\left(\sin \left(\frac{\pi}{2} F(x)\right)\right)^{3}\right) f(x)}{(2 \alpha+\theta \sin (\pi F(x)))^{2}} .
$$


Hence, the associated hrf is

$$
h(x)=\frac{\pi \alpha\left(\alpha \cos \left(\frac{\pi}{2} F(x)\right)+\theta\left(\sin \left(\frac{\pi}{2} F(x)\right)\right)^{3}\right) f(x)}{2 q(x)},
$$

where

$$
\begin{aligned}
q(x) & =\left(\alpha+\theta \cos \left(\frac{\pi}{2} F(x)\right) \sin \left(\frac{\pi}{2} F(x)\right)\right)\left(\alpha-\alpha \sin \left(\frac{\pi}{2} F(x)\right)+\theta \cos \left(\frac{\pi}{2} F(x)\right) \sin \left(\frac{\pi}{2} F(x)\right)\right) \\
& =\frac{1}{4}(2 \alpha+\theta \sin (\pi F(x)))\left(2 \alpha\left(1-\sin \left(\frac{\pi}{2} F(x)\right)\right)+\theta \sin (\pi F(x))\right) .
\end{aligned}
$$

Table 1 presents new cdfs characterizing new distributions arising from the CS1 transformation by considering well-known cdfs for $F(x)$. 


\begin{tabular}{|c|c|c|}
\hline Distribution & $\operatorname{cdf} F(x)$ & $\operatorname{cdf} G(x)$ of the CS1 transformation \\
\hline Uniform $(a, b)$ & $\frac{x-a}{b-a} \mathbf{1}_{[a, b]}(x)+\mathbf{1}_{] b,+\infty[}(x)$ & $\frac{2 \alpha \sin \left(\frac{\pi(x-a)}{2(b-a)}\right)}{2 \alpha+\theta \sin \left(\frac{\pi(x-a)}{b-a}\right)} \mathbf{1}_{[a, b]}(x)+\mathbf{1}_{] b,+\infty[}(x)$ \\
\hline Triangular $(a)$ & $\left(1-\frac{(x-a)^{2}}{a^{2}}\right) \mathbf{1}_{[0, a]}(x)+\mathbf{1}_{] a,+\infty[}(x)$ & $\frac{2 \alpha \cos \left(\frac{\pi(x-a)^{2}}{2 a^{2}}\right)}{2 \alpha+\theta \sin \left(\frac{\pi(x-a)^{2}}{a^{2}}\right)} \mathbf{1}_{[0, a]}(x)+\mathbf{1}_{] a,+\infty[}(x)$ \\
\hline $\operatorname{Exponential}(\lambda)$ & $\left(1-e^{-\frac{x}{\lambda}}\right) \mathbf{1}_{[0,+\infty[}(x)$ & $\frac{2 \alpha \cos \left(\frac{\pi}{2} e^{-\frac{x}{\lambda}}\right)}{2 \alpha+\theta \sin \left(\pi e^{-\frac{x}{\lambda}}\right)} \mathbf{1}_{[0,+\infty[}(x)$ \\
\hline Rayleigh $\left(\sigma^{2}\right)$ & $\left(1-e^{-\frac{x^{2}}{2 \sigma^{2}}}\right) \mathbf{1}_{[0,+\infty[}(x)$ & $\frac{2 \alpha \cos \left(\frac{\pi}{2} e^{-\frac{x^{2}}{2 \sigma^{2}}}\right)}{2 \alpha+\theta \sin \left(\pi e^{-\frac{x^{2}}{2 \sigma^{2}}}\right)} \mathbf{1}_{[0,+\infty[}(x)$ \\
\hline $\operatorname{Normal}\left(\mu, \sigma^{2}\right)$ & $\Phi(x)=\int_{-\infty}^{x} \frac{1}{\sqrt{2 \pi \sigma^{2}}} e^{-\frac{(t-\mu)^{2}}{2 \sigma^{2}}} d t$ & $\frac{2 \alpha \sin \left(\frac{\pi}{2} \Phi(x)\right)}{2 \alpha+\theta \sin (\pi \Phi(x))}$ \\
\hline $\operatorname{Gumbel}(\mu, \beta)$ & $e^{-e^{\frac{\mu-x}{\beta}}}$ & $\frac{2 \alpha \sin \left(\frac{\pi}{2} e^{-e^{\frac{\mu-x}{\beta}}}\right)}{2 \alpha+\theta \sin \left(\pi e^{-e^{\frac{\mu-x}{\beta}}}\right)}$ \\
\hline $\operatorname{Logistic}(\mu, s)$ & $\frac{1}{1+e^{-\frac{x-\mu}{s}}}$ & $\frac{2 \alpha \sin \left(\frac{\pi}{2\left(1+e^{-\frac{x-\mu}{s}}\right)}\right)}{2 \alpha+\theta \sin \left(\frac{\pi}{1+e^{-\frac{x-\mu}{s}}}\right)}$ \\
\hline $\operatorname{Cauchy}\left(x_{0}, a\right)$ & $\frac{1}{\pi} \arctan \left(\frac{x-x_{0}}{a}\right)+\frac{1}{2}$ & $\frac{\sqrt{2} \alpha\left(\sin \left(\frac{1}{2} \arctan \left(\frac{x-x_{0}}{a}\right)\right)+\cos \left(\frac{1}{2} \arctan \left(\frac{x-x_{0}}{a}\right)\right)\right)}{2 \alpha+\theta \cos \left(\arctan \left(\frac{x-x_{0}}{a}\right)\right)}$ \\
\hline Arcsine & $\frac{2}{\pi} \arcsin (\sqrt{x}) \mathbf{1}_{[0,1]}(x)+\mathbf{1}_{] 1,+\infty)}(x)$ & $\frac{\alpha \sqrt{x}}{\alpha+\theta \sqrt{x(1-x)}} \mathbf{1}_{[0,1]}(x)+\mathbf{1}_{] 1,+\infty)}(x)$ \\
\hline $\operatorname{Pareto}\left(x_{m}, k\right)$ & $\left(1-\left(\frac{x_{m}}{x}\right)^{k}\right) \mathbf{1}_{\left[x_{m},+\infty[\right.}(x)$ & $\frac{2 \alpha \cos \left(\frac{\pi}{2}\left(\frac{x_{m}}{x}\right)^{k}\right)}{2 \alpha+\theta \sin \left(\pi\left(\frac{x_{m}}{x}\right)^{k}\right)} \mathbf{1}_{\left[x_{m},+\infty[\right.}(x)$ \\
\hline
\end{tabular}

Table 1: Some cdfs using the CS1 transformation

Proposition 2 below presents a decomposition of the expectation of a function of a random variable having the cdf given by (2).

Proposition 2. Let $X$ be a random variable having the cdf given by $(2)$ and $d(x)$ be a function on $\mathbb{R}$. Then we have

$$
\mathbb{E}(d(X))=\sum_{k=0}^{+\infty} \sum_{\ell=0}^{k} b_{k, \ell} u_{\ell}
$$

where

$$
b_{k, \ell}=2 \pi \alpha \frac{\theta^{k}}{(2 \alpha+\theta)^{k+2}}(k+1)\left(\begin{array}{l}
k \\
\ell
\end{array}\right)(-1)^{\ell}
$$

and

$$
u_{\ell}=\int_{-\infty}^{+\infty}\left(\alpha \cos \left(\frac{\pi}{2} F(x)\right)+\theta\left(\sin \left(\frac{\pi}{2} F(x)\right)\right)^{3}\right)(\sin (\pi F(x)))^{\ell} d(x) f(x) d x
$$


provided that $u_{\ell}$ exist.

The proof of Proposition 2 is given in Appendix. Naturally, $\mathbb{E}(d(X))$ can be useful to determine moments (by taking $d(x)=x^{r}, r \geq 0$ ), characteristic function (by taking $d(x)=e^{i t x}, t \in \mathbb{R}$ )...

Note that $u_{\ell}$ can be expressed as $u_{\ell}=\int_{0}^{1}\left(\alpha \cos \left(\frac{\pi}{2} x\right)+\theta\left(\sin \left(\frac{\pi}{2} x\right)\right)^{3}\right)(\sin (\pi x))^{\ell} d\left(F^{-1}(x)\right) d x$. The complexity in the calculus of $u_{\ell}$ mainly depends on the nature of $d(x), F(x)$ and $f(x)$.

Introduction of the $C S 1_{E}(\alpha, \theta, \lambda)$ distribution. Let us now focus our attention on the CS1 transformation in the case where $F(x)$ is the cdf of the exponential distribution with parameter $\lambda>0$. We call this distribution as the $C S 1_{E}(\alpha, \theta, \lambda)$ distribution for frequently used purposed. As noted in Table 1, using trigonometric formulas, the associated cdf is

$$
G(x)=\frac{2 \alpha \sin \left(\frac{\pi}{2}\left(1-e^{-\frac{x}{\lambda}}\right)\right)}{2 \alpha+\theta \sin \left(\pi\left(1-e^{-\frac{x}{\lambda}}\right)\right)} \mathbf{1}_{[0,+\infty)}(x)=\frac{2 \alpha \cos \left(\frac{\pi}{2} e^{-\frac{x}{\lambda}}\right)}{2 \alpha+\theta \sin \left(\pi e^{-\frac{x}{\lambda}}\right)} \mathbf{1}_{[0,+\infty)}(x) .
$$

Note that, taking $\theta=0$ and $\lambda=\frac{1}{\nu}$, we obtain the $S S_{E}(\nu)$ distribution introduced by [14].

Using the same mathematical arguments, the associated pdf and hzf are, respectively, given by

$$
g(x)=\frac{2 \pi \alpha\left(\alpha \sin \left(\frac{\pi}{2} e^{-\frac{x}{\lambda}}\right)+\theta\left(\cos \left(\frac{\pi}{2} e^{-\frac{x}{\lambda}}\right)\right)^{3}\right) e^{-\frac{x}{\lambda}}}{\lambda\left(2 \alpha+\theta \sin \left(\pi e^{-\frac{x}{\lambda}}\right)\right)^{2}} \mathbf{1}_{[0,+\infty)}(x)
$$

and

$$
h(x)=\frac{2 \pi \alpha\left(\alpha \sin \left(\frac{\pi}{2} e^{-\frac{x}{\lambda}}\right)+\theta\left(\cos \left(\frac{\pi}{2} e^{-\frac{x}{\lambda}}\right)\right)^{3}\right) e^{-\frac{x}{\lambda}}}{\lambda\left(2 \alpha+\theta \sin \left(\pi e^{-\frac{x}{\lambda}}\right)\right)\left(2 \alpha\left(1-\cos \left(\frac{\pi}{2} e^{-\frac{x}{\lambda}}\right)\right)+\theta \sin \left(\pi e^{-\frac{x}{\lambda}}\right)\right)} \mathbf{1}_{[0,+\infty)}(x) .
$$

To show the flexibility of this distributions, we provide some graphs. The associated cdf, pdf and hrf are presented in Figures 1, 2 and 3, where various shapes are observed and showing symmetries and skewness. Applicability of the $C S 1_{E}(\alpha, \theta, \lambda)$ distribution will be explored in Section 6 . 


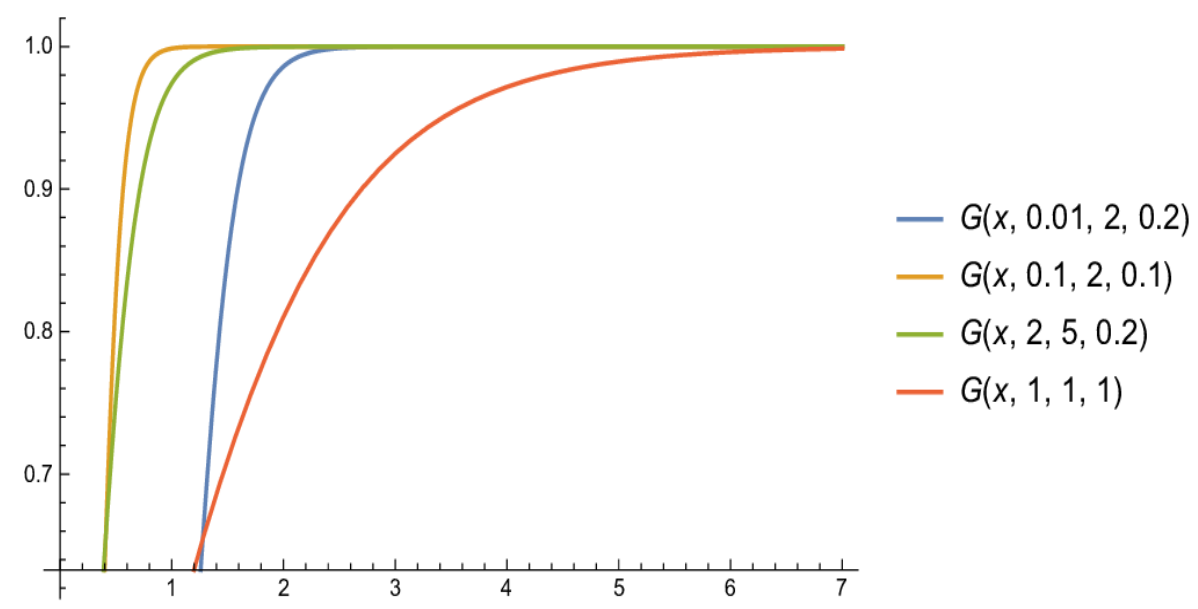

Figure 1: Some cdfs $G(x)=G(x, \alpha, \theta, \lambda)$ (4) associated to the distribution $C S 1_{E}(\alpha, \theta, \lambda)$.

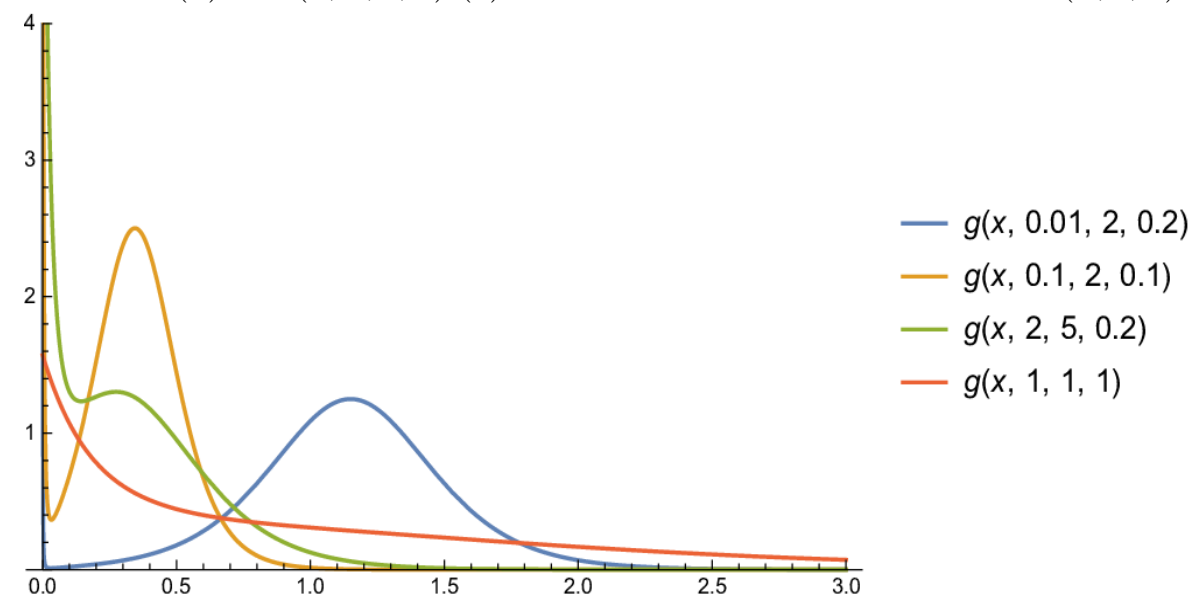

Figure 2: Some pdfs $g(x)=g(x, \alpha, \theta, \lambda)(5)$ associated to the distribution $C S 1_{E}(\alpha, \theta, \lambda)$.

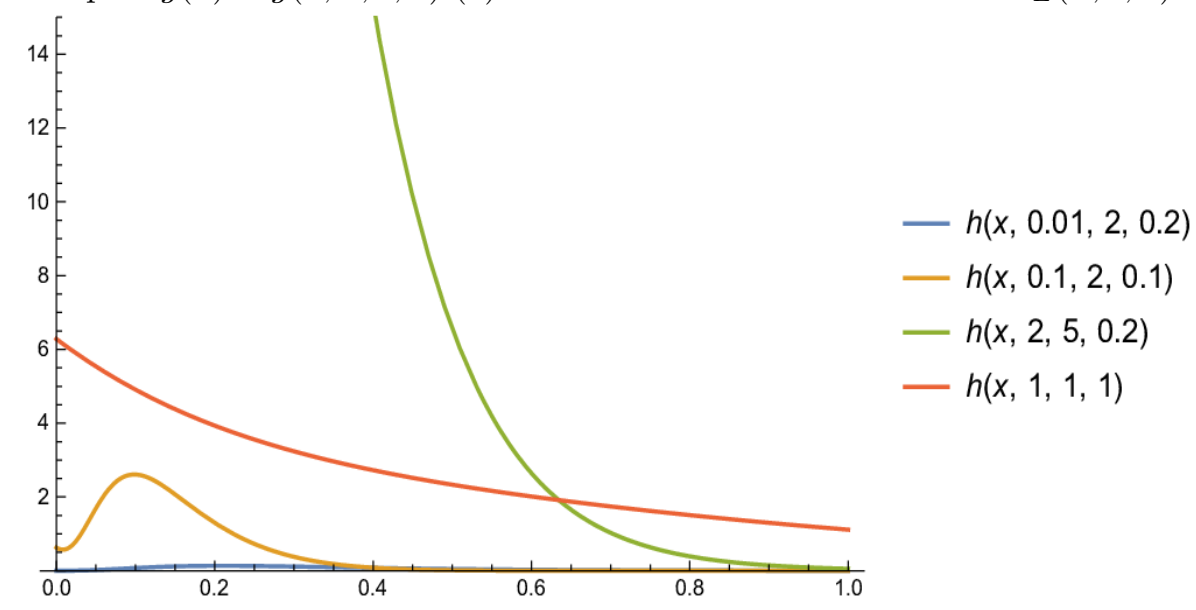

Figure 3: Some hrfs $h(x)=h(x, \alpha, \theta, \lambda)(6)$ associated to the distribution $C S 1_{E}(\alpha, \theta, \lambda)$. 


\section{The CS2 transformation}

Let us now consider another particular case of the CS transformation. Taking $\alpha=0, \beta>0, \gamma>0$ and $\theta=0, G(x)$ becomes the following function :

$$
G(x)=\frac{\gamma \sin \left(\frac{\pi}{2} F(x)\right)}{\beta \cos \left(\frac{\pi}{2} F(x)\right)+\gamma \sin \left(\frac{\pi}{2} F(x)\right)} .
$$

An alternative expression of $G(x)$ is given by using the survival function $S(x)$ defined by $S(x)=$ $1-F(x)$ and standard trigonometric formulas:

$$
G(x)=\frac{\gamma \sin \left(\frac{\pi}{2}(1-S(x))\right)}{\beta \cos \left(\frac{\pi}{2}(1-S(x))\right)+\gamma \sin \left(\frac{\pi}{2}(1-S(x))\right)}=\frac{\gamma \cos \left(\frac{\pi}{2} S(x)\right)}{\beta \sin \left(\frac{\pi}{2} S(x)\right)+\gamma \cos \left(\frac{\pi}{2} S(x)\right)} .
$$

In this case, observe that the associated pdf and hrf have the forms :

$$
g(x)=\frac{\pi \gamma \beta f(x)}{2\left(\beta \cos \left(\frac{\pi}{2} F(x)\right)+\gamma \sin \left(\frac{\pi}{2} F(x)\right)\right)^{2}}
$$

and

$$
h(x)=\frac{\pi \gamma f(x)}{2\left(\beta \cos \left(\frac{\pi}{2} F(x)\right)+\gamma \sin \left(\frac{\pi}{2} F(x)\right)\right) \cos \left(\frac{\pi}{2} F(x)\right)} .
$$

Table 2 presents new cdfs characterizing new distributions arising from the CS2 transformation by considering well-known cdfs for $F(x)$. 


\begin{tabular}{|c|c|c|}
\hline Distribution & $\operatorname{cdf} F(x)$ & $\operatorname{cdf} G(x)$ of the CS2 transformation \\
\hline Uniform $(a, b)$ & $\frac{x-a}{b-a} \mathbf{1}_{[a, b]}(x)+\mathbf{1}_{] b,+\infty[}(x)$ & $\frac{\gamma \sin \left(\frac{\pi(x-a)}{2(b-a)}\right)}{\beta \cos \left(\frac{\pi(x-a)}{2(b-a)}\right)+\gamma \sin \left(\frac{\pi(x-a)}{2(b-a)}\right)} \mathbf{1}_{[a, b]}(x)+\mathbf{1}_{] b,+\infty[}(x)$ \\
\hline Triangular $(a)$ & $\left(1-\frac{(x-a)^{2}}{a^{2}}\right) \mathbf{1}_{[0, a]}(x)+\mathbf{1}_{] a,+\infty[}(x)$ & $\frac{\gamma \cos \left(\frac{\pi(x-a)^{2}}{2 a^{2}}\right)}{\beta \sin \left(\frac{\pi(x-a)^{2}}{2 a^{2}}\right)+\gamma \cos \left(\frac{\pi(x-a)^{2}}{2 a^{2}}\right)} \mathbf{1}_{[0, a]}(x)+\mathbf{1}_{] a,+\infty[}(x)$ \\
\hline $\operatorname{Exponential}(\lambda)$ & $\left(1-e^{-\frac{x}{\lambda}}\right) \mathbf{1}_{[0,+\infty[}(x)$ & $\frac{\gamma \cos \left(\frac{\pi}{2} e^{-\frac{x}{\lambda}}\right)}{\beta \sin \left(\frac{\pi}{2} e^{-\frac{x}{\lambda}}\right)+\gamma \cos \left(\frac{\pi}{2} e^{-\frac{x}{\lambda}}\right)} \mathbf{1}_{[0,+\infty[}(x)$ \\
\hline Rayleigh $\left(\sigma^{2}\right)$ & $\left(1-e^{-\frac{x^{2}}{2 \sigma^{2}}}\right) \mathbf{1}_{[0,+\infty[}(x)$ & $\frac{\gamma \sin \left(\frac{\pi}{2} e^{-\frac{x^{2}}{2 \sigma^{2}}}\right)+\gamma \cos \left(\frac{\pi}{2} e^{-\frac{x^{2}}{2 \sigma^{2}}}\right)}{\mathbf{1}_{[0,+\infty[}(x)}$ \\
\hline $\operatorname{Normal}\left(\mu, \sigma^{2}\right)$ & $\Phi(x)=\int_{-\infty}^{x} \frac{1}{\sqrt{2 \pi \sigma^{2}}} e^{-\frac{(t-\mu)^{2}}{2 \sigma^{2}}} d t$ & $\frac{\gamma \sin \left(\frac{\pi}{2} \Phi(x)\right)}{\beta \cos \left(\frac{\pi}{2} \Phi(x)\right)+\gamma \sin \left(\frac{\pi}{2} \Phi(x)\right)}$ \\
\hline $\operatorname{Gumbel}(\mu, \beta)$ & $e^{-e^{\frac{\mu-x}{\beta}}}$ & $\frac{1}{\beta \cos \left(\frac{\pi}{2} e^{-e^{\frac{\mu-x}{\beta}}}\right)+\gamma \sin \left(\frac{\pi}{2} e^{-e^{\frac{\mu-x}{\beta}}}\right)}$ \\
\hline $\operatorname{Logistic}(\mu, s)$ & $\frac{1}{1+e^{-\frac{x-\mu}{s}}}$ & $\frac{\left(2\left(1+e^{-\frac{x-\mu}{s}}\right)\right)}{\beta \cos \left(\frac{\pi}{2\left(1+e^{-\frac{x-\mu}{s}}\right)}\right)+\gamma \sin \left(\frac{\pi}{2\left(1+e^{-\frac{x-\mu}{s}}\right)}\right)}$ \\
\hline $\operatorname{Cauchy}\left(x_{0}, a\right)$ & $\frac{1}{\pi} \arctan \left(\frac{x-x_{0}}{a}\right)+\frac{1}{2}$ & $\frac{\gamma\left(\sin \left(\frac{1}{2} \arctan \left(\frac{x-x_{0}}{a}\right)\right)+\cos \left(\frac{1}{2} \arctan \left(\frac{x-x_{0}}{a}\right)\right)\right)}{(\gamma-\beta) \sin \left(\frac{1}{2} \arctan \left(\frac{x-x_{0}}{a}\right)\right)+(\gamma+\beta) \cos \left(\frac{1}{2} \arctan \left(\frac{x-x_{0}}{a}\right)\right.}$ \\
\hline Arcsine & $\frac{2}{\pi} \arcsin (\sqrt{x}) \mathbf{1}_{[0,1]}(x)+\mathbf{1}_{] 1,+\infty)}(x)$ & $\frac{\gamma \sqrt{x}}{\beta \sqrt{1-x}+\gamma \sqrt{x}} \mathbf{1}_{[0,1]}(x)+\mathbf{1}_{] 1,+\infty)}(x)$ \\
\hline $\operatorname{Pareto}\left(x_{m}, k\right)$ & $\left(1-\left(\frac{x_{m}}{x}\right)^{k}\right) \mathbf{1}_{\left[x_{m},+\infty[\right.}(x)$ & $\frac{\gamma \cos \left(\frac{\pi}{2}\left(\frac{x_{m}}{x}\right)^{k}\right)}{\beta \sin \left(\frac{\pi}{2}\left(\frac{x_{m}}{x}\right)^{k}\right)+\gamma \cos \left(\frac{\pi}{2}\left(\frac{x_{m}}{x}\right)^{k}\right)} \mathbf{1}_{\left[x_{m},+\infty[\right.}(x)$ \\
\hline
\end{tabular}

Table 2: Some cdfs using the CS2 transformation

Proposition 3 below presents a decomposition of the expectation of a function of a random variable having the cdf given by (2).

Proposition 3. Let $X$ be a random variable having the cdf given by $(7)$ and $d(x)$ be a function on $\mathbb{R}$. Then we have

$$
\mathbb{E}(d(X))=\sum_{k=0}^{+\infty} \sum_{\ell=0}^{k} \sum_{m=0}^{\ell} \sum_{s=0}^{k-\ell} \sum_{t=0}^{+\infty} c_{k, \ell, m, s, t} v_{s, t}
$$

where

$$
c_{k, \ell, m, s, t}=\frac{\pi \gamma \beta}{2(\alpha+\beta)^{2}}(k+1)\left(\begin{array}{c}
k \\
\ell
\end{array}\right)\left(\begin{array}{c}
\ell \\
m
\end{array}\right)\left(\begin{array}{c}
k-\ell \\
s
\end{array}\right)\left(\begin{array}{c}
m / 2 \\
t
\end{array}\right) \frac{\beta^{\ell}}{(\alpha+\beta)^{\ell}}(-1)^{m+s+t} \frac{\alpha^{k-\ell}}{(\alpha+\beta)^{k-\ell}}
$$


and

$$
v_{s, t}=\int_{-\infty}^{+\infty}\left(\sin \left(\frac{\pi}{2} F(x)\right)\right)^{s+2 t} d(x) f(x) d x
$$

provided that $v_{s, t}$ exist.

The proof of Proposition 3 is given in Appendix. This can be useful to determine moments, characteristic function...

Note that $v_{s, t}$ can be expressed as $v_{s, t}=\int_{0}^{1}\left(\sin \left(\frac{\pi}{2} x\right)\right)^{s+2 t} d\left(F^{-1}(x)\right) d x$. The difficulty in the calculus of $v_{s, t}$ mainly depends on the nature of $d(x), F(x)$ and $f(x)$.

Introduction of the $C S 2_{E}(\beta, \gamma, \lambda)$ distribution. Let us now focus our attention on the CS2 transformation in the case where $F(x)$ is the cdf of the exponential distribution with parameter $\lambda>0: F(x)=\left(1-e^{-\frac{x}{\lambda}}\right) \mathbf{1}_{[0,+\infty)}(x)$. We call this distribution as the $C S 2_{E}(\beta, \gamma, \lambda)$ distribution for frequently used purposed. As noted in Table 2, using trigonometric formula, we obtain

$$
\begin{aligned}
G(x) & =\frac{\gamma \sin \left(\frac{\pi}{2}\left(1-e^{-\frac{x}{\lambda}}\right)\right)}{\beta \cos \left(\frac{\pi}{2}\left(1-e^{-\frac{x}{\lambda}}\right)\right)+\gamma \sin \left(\frac{\pi}{2}\left(1-e^{-\frac{x}{\lambda}}\right)\right)} \mathbf{1}_{[0,+\infty)}(x) \\
& =\frac{\gamma \cos \left(\frac{\pi}{2} e^{-\frac{x}{\lambda}}\right)}{\beta \sin \left(\frac{\pi}{2} e^{-\frac{x}{\lambda}}\right)+\gamma \cos \left(\frac{\pi}{2} e^{-\frac{x}{\lambda}}\right)} \mathbf{1}_{[0,+\infty)}(x) .
\end{aligned}
$$

Similar mathematical arguments yields the associated pdf and hrf :

$$
g(x)=\frac{\pi \gamma \beta e^{-\frac{x}{\lambda}}}{2 \lambda\left(\beta \sin \left(\frac{\pi}{2} e^{-\frac{x}{\lambda}}\right)+\gamma \cos \left(\frac{\pi}{2} e^{-\frac{x}{\lambda}}\right)\right)^{2}} \mathbf{1}_{[0,+\infty)}(x)
$$

and

$$
h(x)=\frac{\pi \gamma e^{-\frac{x}{\lambda}}}{2 \lambda\left(\beta \sin \left(\frac{\pi}{2} e^{-\frac{x}{\lambda}}\right)+\gamma \cos \left(\frac{\pi}{2} e^{-\frac{x}{\lambda}}\right)\right) \sin \left(\frac{\pi}{2} e^{-\frac{x}{\lambda}}\right)} \mathbf{1}_{[0,+\infty)}(x) .
$$

In order to illustrate the nature of the $C S 2_{E}(\beta, \gamma, \lambda)$ distribution, graphs of some cdfs, pdfs and hrfs are presented in Figures 4, 5 and 6 . They show various shapes of those functions. 


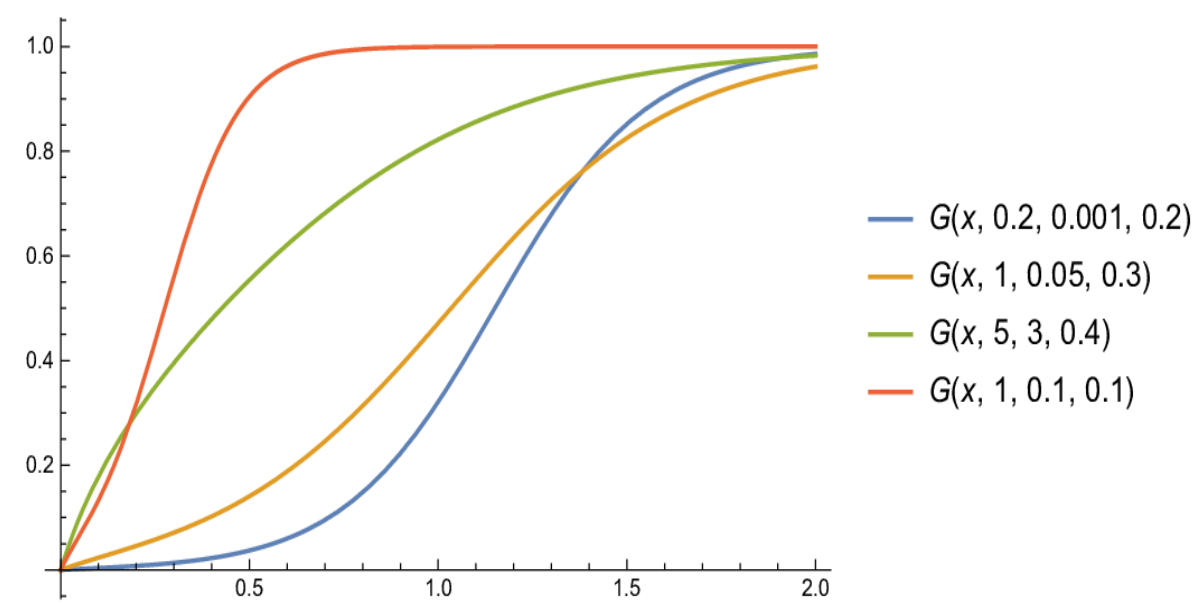

Figure 4: Some cdfs $G(x)=G(x, \beta, \gamma, \lambda)(9)$ associated to the distribution $C S 2_{E}(\beta, \gamma, \lambda)$.

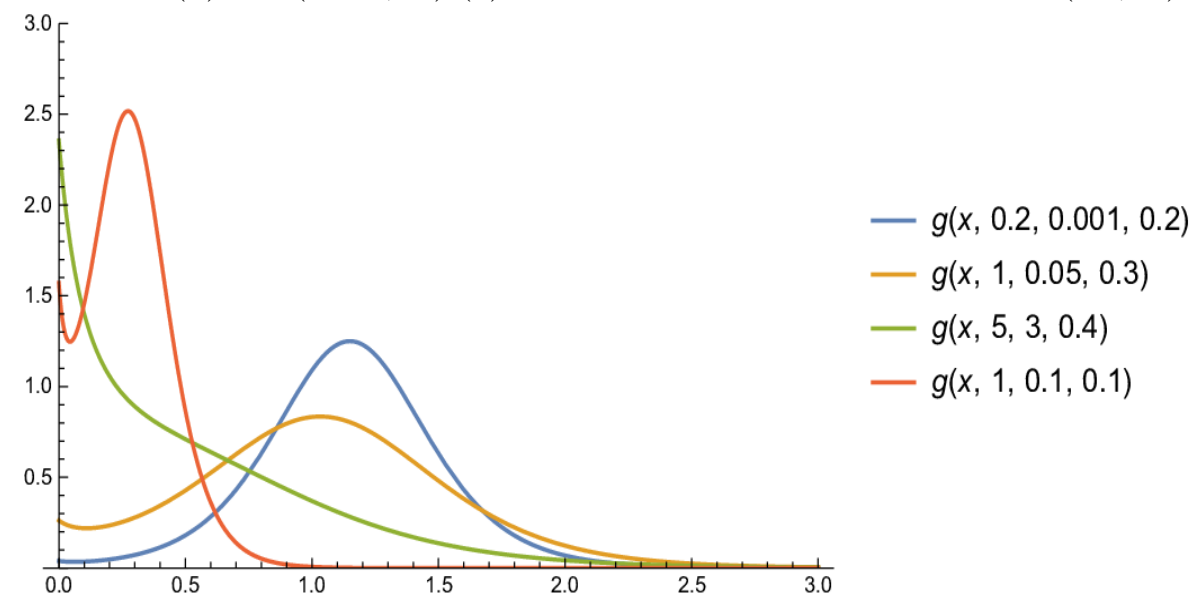

Figure 5: Some pdfs $g(x)=g(x, \beta, \gamma, \lambda)(10)$ associated to the distribution $C S 2_{E}(\beta, \gamma, \lambda)$.

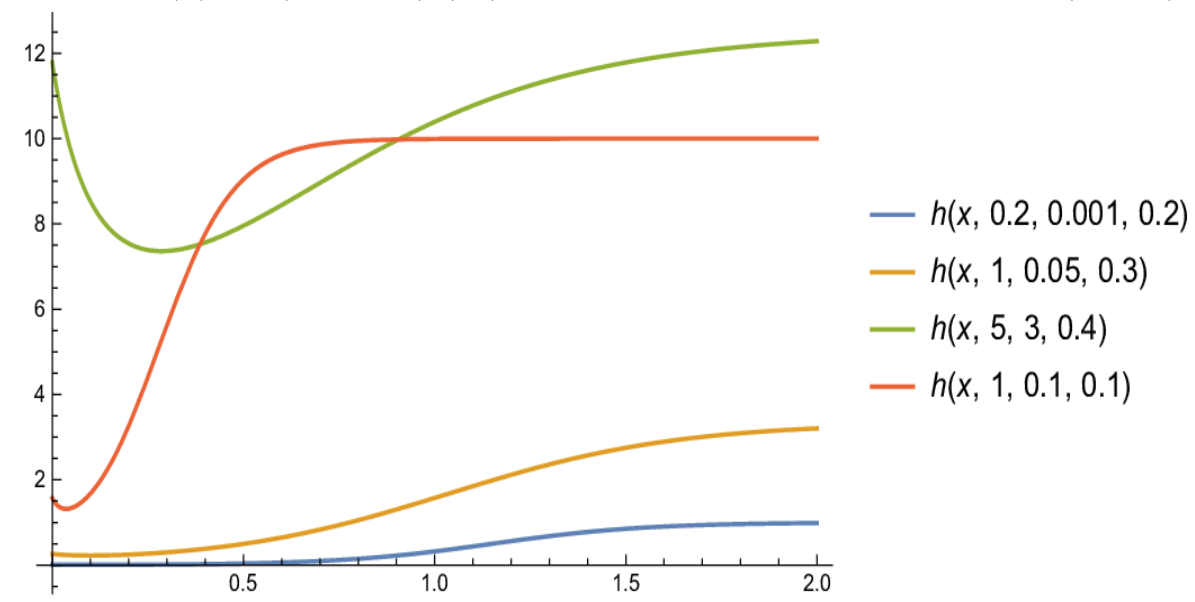

Figure 6: Some hrfs $h(x)=h(x, \beta, \gamma, \lambda)(11)$ associated to the distribution $C S 2_{E}(\beta, \gamma, \lambda)$. 


\section{Maximum Likelihood Estimation and simulation}

\subsection{Maximum likelihood estimation:}

Let $X_{1}, X_{2}, \ldots, X_{n}$ be a random sample from the $C S 1_{E}(\alpha, \theta, \lambda)$ distribution with parameter vector $\Theta=(\alpha, \theta, \lambda)$ and $x_{1}, x_{2}, \ldots, x_{n}$ are the observed values, then joint probability function of $X_{1}, X_{2}, \ldots, X_{n}$ as a log-likelihood function can be expressed as

$$
\begin{aligned}
\ell(\Theta) & =n \log \left(\frac{2 \pi \alpha}{\lambda}\right)+\sum_{i=1}^{n} \log \left(\alpha \sin \left(\frac{\pi}{2} e^{-\frac{x_{i}}{\lambda}}\right)+\theta\left(\cos \left(\frac{\pi}{2} e^{-\frac{x_{i}}{\lambda}}\right)\right)^{3}\right)-\frac{1}{\lambda} \sum_{i=1}^{n} x_{i} \\
& -2 \sum_{i=1}^{n} \log \left(2 \alpha+\theta \sin \left(\pi e^{-\frac{x_{i}}{\lambda}}\right)\right) .
\end{aligned}
$$

The associated nonlinear $\log$-likelihood equations $\frac{\partial \ell(\Theta)}{\partial \Theta}=0$ are given by

$$
\begin{gathered}
\frac{\partial \ell(\Theta)}{\partial \alpha}=\frac{n}{\alpha}+\sum_{i=1}^{n} \frac{\sin \left(\frac{\pi}{2} e^{-\frac{x_{i}}{\lambda}}\right)}{\alpha \sin \left(\frac{\pi}{2} e^{-\frac{x_{i}}{\lambda}}\right)+\theta\left(\cos \left(\frac{\pi}{2} e^{-\frac{x_{i}}{\lambda}}\right)\right)^{3}}-4 \sum_{i=1}^{n} \frac{1}{2 \alpha+\theta \sin \left(\pi e^{-\frac{x_{i}}{\lambda}}\right)}=0 \\
\frac{\partial \ell(\Theta)}{\partial \theta}=\sum_{i=1}^{n} \frac{\left(\cos \left(\frac{\pi}{2} e^{-\frac{x_{i}}{\lambda}}\right)\right)^{3}}{\alpha \sin \left(\frac{\pi}{2} e^{-\frac{x_{i}}{\lambda}}\right)+\theta\left(\cos \left(\frac{\pi}{2} e^{-\frac{x_{i}}{\lambda}}\right)\right)^{3}}-2 \sum_{i=1}^{n} \frac{\sin \left(\pi e^{-\frac{x_{i}}{\lambda}}\right)}{2 \alpha+\theta \sin \left(\pi e^{-\frac{x_{i}}{\lambda}}\right)}=0
\end{gathered}
$$

and

$$
\begin{aligned}
\frac{\partial \ell(\Theta)}{\partial \lambda} & =-\frac{n}{\lambda}+\sum_{i=1}^{n} \frac{\pi x_{i} e^{-\frac{x_{i}}{\lambda}}\left(\alpha \cos \left(\frac{\pi}{2} e^{-\frac{x_{i}}{\lambda}}\right)-3 \theta\left(\cos \left(\frac{\pi}{2} e^{-\frac{x_{i}}{\lambda}}\right)\right)^{2} \sin \left(\frac{\pi}{2} e^{-\frac{x_{i}}{\lambda}}\right)\right)}{2 \lambda^{2}\left(\alpha \sin \left(\frac{\pi}{2} e^{-\frac{x_{i}}{\lambda}}\right)+\theta\left(\cos \left(\frac{\pi}{2} e^{-\frac{x_{i}}{\lambda}}\right)\right)^{3}\right)} \\
& -2 \sum_{i=1}^{n} \frac{\pi x_{i} e^{-\frac{x_{i}}{\lambda}} \cos \left(\pi e^{-\frac{x_{i}}{\lambda}}\right)}{\lambda^{2}\left(2 \alpha+\theta \sin \left(\pi e^{-\frac{x_{i}}{\lambda}}\right)\right)}=0 .
\end{aligned}
$$

By solving these three equations simultaneously, we obtain the maximum likelihood estimators of the parameters.

\subsection{Simulation study}

A general form to generate a random variable $X$ from the $C S 1_{E}(\alpha, \theta, \lambda)$ and $C S 2_{E}(\beta, \gamma, \lambda)$ distributions is to generate the values $x$ from the proposed models with parameters $\theta, \alpha, \lambda$ and $\beta, \gamma, \lambda$ by using the Mathematica 8.0 computational package. A complete simulation analysis of the CS1 and CS2 distributions was carried out by generating 1000 samples for each of triplet $(\theta, \alpha, \lambda)$ and $(\beta, \gamma, \lambda)$ with $n=25,50,100$. The analysis computes the following values: - Average bias of the simulated estimates:

$$
\frac{1}{n} \sum_{i=1}^{n}\left(\Theta^{\star}-\Theta\right)
$$

where $\Theta^{\star}=(\hat{\alpha}, \hat{\theta}, \hat{\lambda})$ are the MLEs of the $C S 1_{E}(\alpha, \theta, \lambda)$ distribution and $\Theta^{\star}=(\hat{\beta}, \hat{\gamma}, \hat{\lambda})$. are the MLEs of the $C S 2_{E}(\beta, \gamma, \lambda)$ distribution, respectively.

- Average mean square error (MSE) of the simulated estimates:

$$
\frac{1}{n} \sum_{i=1}^{n}\left(\Theta^{\star}-\Theta\right)^{2}
$$


Table 3 concerns the $C S 1_{E}(\alpha, \theta, \lambda)$ distribution. It shows the average bias and average MSE of the estimates for different values of $\alpha, \theta, \lambda$. A downward bias for $\hat{\alpha}$ and $\hat{\lambda}$ and upward bias for $\hat{\theta}$ is observed when $\alpha, \theta$ and $\lambda$ less than one, similar results are also observed in MSE of the estimates. However when the true value of parameter $\lambda$ and $\theta$ becomes greater than one and $\alpha$ less than one the downward biases for $\hat{\alpha}$ and $\hat{\lambda}$ is worth mentioning. Moreover, if all the parameters exceeds one a downward bias is only for $\hat{\alpha}$ and $\hat{\theta}$ and $\hat{\lambda}$ upward bias. In addition the biases and MSE decreases as sample size increases.

Similarly Table 4 portrays the average bias and average MSE of the $C S 2_{E}(\beta, \gamma, \lambda)$ parameters estimates. During simulation study, it is noticed that MLE $\hat{\gamma}$ fluctuate between 0.0009 to 6 while the $\hat{\beta}$ may also vary from 0.25 to 35 . However, the $\hat{\lambda}$ remains unchanged. Moreover, it is also noted that when parameters are greater than one a downward bias in $\hat{\gamma}$ and $\hat{\beta}$ is observed and when all parameters values are less than one an upward bias can be seen in all estimates, also bias and MSE becomes smaller as sample size increase. However, for $\lambda>1$ and for $\gamma, \beta<1$ a downward bias with decreasing attitude in both bias and MSE is observed.

\begin{tabular}{cccccccc}
\hline Parameter & Sample Size & $\operatorname{Bias}(\hat{\alpha})$ & $\operatorname{Bias}(\hat{\theta})$ & $\operatorname{Bias}(\hat{\lambda})$ & $\operatorname{MSE}(\hat{\alpha})$ & $\operatorname{MSE}(\hat{\theta})$ & $\operatorname{MSE}(\hat{\lambda})$ \\
\hline & $n=25$ & -0.2856 & 17.0311 & -0.2198 & 0.0427 & 290.9392 & 0.0496 \\
& $n=50$ & -0.2641 & 16.30518 & -0.2048 & 0.0392 & 266.3227 & 0.0461 \\
$\alpha=0.7221, \theta=0.3498, \lambda=0.4123$ & $n=100$ & -0.2145 & 16.1199 & -0.2032 & 0.0348 & 261.0002 & 0.0449 \\
\hline & $n=25$ & -0.0017 & 15.0352 & -1.1195 & 0.0164 & 246.9911 & 1.3995 \\
$\alpha=0.5212, \theta=1.2131, \lambda=1.5317$ & $n=100$ & -0.0011 & 15.0108 & -1.1094 & 0.0125 & 238.2420 & 1.2990 \\
\hline & $n=25$ & -0.4764 & -3.0304 & 3.8484 & 0.2329 & 9.1843 & 14.8262 \\
$\alpha=1.3247, \theta=3.7904, \lambda=2.3683$ & $n=100$ & -0.4271 & -3.0171 & 3.8478 & 0.1873 & 9.1166 & 14.7576 \\
\hline
\end{tabular}

Table 3: Average bias and MSE values from simulation of the $C S 1_{E}(\alpha, \theta, \lambda)$ distribution

\begin{tabular}{|c|c|c|c|c|c|c|c|}
\hline Parameter & Sample Size & $\operatorname{Bias}(\hat{\gamma})$ & $\operatorname{Bias}(\hat{\beta})$ & $\operatorname{Bias}(\hat{\lambda})$ & $\operatorname{MSE}(\hat{\gamma})$ & $\operatorname{MSE}(\hat{\beta})$ & $\operatorname{MSE}(\hat{\lambda})$ \\
\hline \multirow{3}{*}{$\beta=0.5096, \gamma=1.8465, \lambda=0.7846$} & $n=25$ & -1.4062 & -0.3042 & -0.2323 & 2.1054 & 0.1719 & 0.6437 \\
\hline & $n=50$ & -0.9001 & -0.1929 & -0.2054 & 0.6439 & 0.1583 & 0.3744 \\
\hline & $n=100$ & -0.2839 & -0.1508 & -0.1609 & 0.3578 & 0.1378 & 0.3719 \\
\hline \multirow{3}{*}{$\beta=0.5476, \gamma=0.5324, \lambda=0.5634$} & $n=25$ & 0.9139 & 1.3176 & 0.0479 & 3.1477 & 9.2488 & 0.0473 \\
\hline & $n=50$ & 0.7406 & 1.2017 & 0.0299 & 2.4056 & 5.2761 & 0.0194 \\
\hline & $n=100$ & 0.5277 & 0.6437 & 0.0079 & 1.0018 & 4.0083 & 0.0091 \\
\hline \multirow{3}{*}{$\beta=3.6543, \gamma=2.4537, \lambda=1.6533$} & $n=25$ & -1.9584 & -2.9835 & 0.0200 & 3.9079 & 8.9075 & 0.2275 \\
\hline & $n=50$ & -1.9228 & -2.9809 & 0.0152 & 3.8261 & 8.8341 & 0.1233 \\
\hline & $n=100$ & -1.0185 & -2.0011 & 0.0135 & 3.0921 & 8.0086 & 0.0451 \\
\hline
\end{tabular}

Table 4: Average bias and MSE values from simulation of the $C S 2_{E}(\beta, \gamma, \lambda)$ distribution 


\section{Evaluation tests and real data examples}

In statistical literature, a plethora of distributions exist for life testing experiments. Some of them are suitable in increasing/decreasing failure rate scenario, others are appropriate for bathtub and upside down bath tub shapes and some are for both features. In this context, we studied those distributions which are very strong in their respective area, namely transmuted modified inverse Rayleigh (TMIR) defined by [10], transmuted inverse Weibull (TIW) introduced by [11], new modified Weibull (MW) defined by [15], generalized linear failure rate (GLFR) defined by [18] and single parameter sine transformed distributions proposed by [13]. The pdfs of the competing models are expressed in Table 5 .

\begin{tabular}{cccc} 
Name & Pdf & Authors & Domain \\
\hline TMIR & $g(x)=\left(\alpha+\frac{2 \theta}{x}\right)\left(\frac{1}{x}\right)^{2} e^{-\left(\frac{\alpha}{x}+\frac{\theta}{x^{2}}\right)}\left\{1+\lambda-2 \lambda e^{-\left(\frac{\alpha}{x}+\frac{\theta}{x^{2}}\right)}\right\}$ & Khan and King (2015) [10] & $x>0, \alpha, \theta>0,|\lambda| \leq 1$ \\
TIW & $g(x)=\alpha \theta^{\alpha} x^{-\alpha-1} e^{-\left(\frac{\theta}{x}\right)^{\alpha}}\left\{1+\lambda-2 \lambda e^{-\left(\frac{\theta}{x}\right)^{\alpha}}\right\}$ & Khan et al. (2014) [11] & $x>0, \alpha, \theta>0,|\lambda| \leq 1$ \\
GLFR & $g(x)=\theta(\alpha+\lambda x)\left(1-e^{-\left(\alpha x+0.5 \lambda x^{2}\right)}\right)^{\theta-1} e^{-\left(\alpha x+0.5 \lambda x^{2}\right)}$ & Sarhan and Kundu (2014) $[18]$ & $x>0, \alpha, \theta, \lambda>0$ \\
MW & $g(x)=\theta(\alpha+\lambda x) x^{\alpha-1} e^{\lambda x-\theta x^{\alpha} e^{\lambda x}}$ & Lai et al. (2003) [15] & $x>0, \alpha, \theta, \lambda>0$ \\
ST & $g(x)=\frac{\pi}{2} \beta e^{-\beta x} \sin \left(\frac{\pi}{2} e^{-\beta x}\right)$ & Kumar et al. (2015) $[13]$ & $x>0, \theta>0$ \\
\hline
\end{tabular}

Table 5: The compared distributions to the CS distribution

\subsection{Evaluation tests}

In order to demonstrate the proposed methodology, we consider four different real-world data sets, representing various failure rate pattern like increasing, decreasing, and bathtub shape, compared via the Akaike Information Criterion (AIC), the Corrected Akaike Information criterion (AICc), the Hannan-Quinn Information criterion (HQIC) and the Consistent Akaike Information criterion (CAIC) which are used to select the best model among several models. The definitions of AIC, AICc, HQIC and CAIC are given below:

$$
\begin{gathered}
A I C=2 k-2 \ell(\hat{\Theta}), \quad A I C c=A I C+\frac{2 k(k+1)}{n-k-1}, \\
H Q I C=-2 \ell(\hat{\Theta})+2 k \ln (\ln (n)), \quad C A I C=-2 \ell(\hat{\Theta})+\frac{2 k n}{n-k-1} .
\end{gathered}
$$

Moreover, perfection of competing models is also tested via the Kolmogrov-Simnorov(K-S), the Anderson-Darling $\left(A_{0}^{*}\right)$ and the Cramer Von Misses $\left(\mathrm{W}_{0}^{*}\right)$ statistics. The mathematical expressions for the statistics above are given below

$$
\begin{gathered}
K S=\max \left\{\frac{i}{m}-z_{i}, z_{i}-\frac{i-1}{m}\right\}, \\
A_{0}^{*}=\left(\frac{2.25}{m^{2}}+\frac{0.75}{m}+1\right)\left\{-m-\frac{1}{m} \sum_{i=1}^{m}(2 i-1) \ln \left(z_{i}\left(1-z_{m-i+1}\right)\right)\right\}, \\
W_{0}^{*}=\sum_{i=1}^{m}\left(z_{i}-\frac{2 i-1}{2 m}\right)^{2}+\frac{1}{12 m},
\end{gathered}
$$

where $k$ denotes the number of parameters, $n$ denotes the number of observations, $m$ denotes the number of classes and $z_{i}=c d f\left(x_{i}\right)$, the $x_{i}$ 's being the ordered observations. It is also worth mentioning that we have not only confine our interest in the information criterion and statistics but also adopted the graphical displays, so that the reader can gain a perspective of the various meanings and associated interpretations. Moreover, in many applications there is a qualitative information about the failure rate shape, which can help in selecting a specified model. A hazard may be considered as a dangerous 
event that can lead to an emergency or disaster. A hazard analysis can be performed statistically based on the hazard rate (HR), also known as chance function, failure rate, intensity function, or risk rate, among other names. A nice property of the HR is that it allows us to better characterize the behavior of statistical distributions, and to differentiate models with very similar cdfs. For example, the HR may have several different shapes, such as increasing (IHR), constant, decreasing (DHR), bathtub (BT), inverse bathtub (IBT) approaching a non-null constant, or IBT approaching zero. In this regard, a device called the total time on test (TTT) plot is useful see [2]. Usually the TTT plot is drawn by plotting $T\left(\frac{i}{n}\right)=\frac{\sum_{r=1}^{i} y_{r: n}+(n-i) y_{i: n}}{\sum_{r=1}^{n} y_{r: n}}$ against $\frac{i}{n}$, where $i=1, \ldots, n$ and $y_{r: n}, r=1, \ldots, n$ are the order statistics of the sample. A TTT curve may be concave (convex) is related to the IHR (DHR) class. A concave (convex) and then convex (concave) TTT curve is related to a BT (IBT) HR. Finally, a TTT curve expressed by a straight line corresponds to the exponential distribution. The TTT plots for the current data sets are displayed in Figure 10, which reveal increasing hrfs in both cases. Therefore, these plots indicate the appropriateness of the new family to fit these data, since its special models can present increasing, decreasing, bathtub and upside-down bathtub hazard functions.

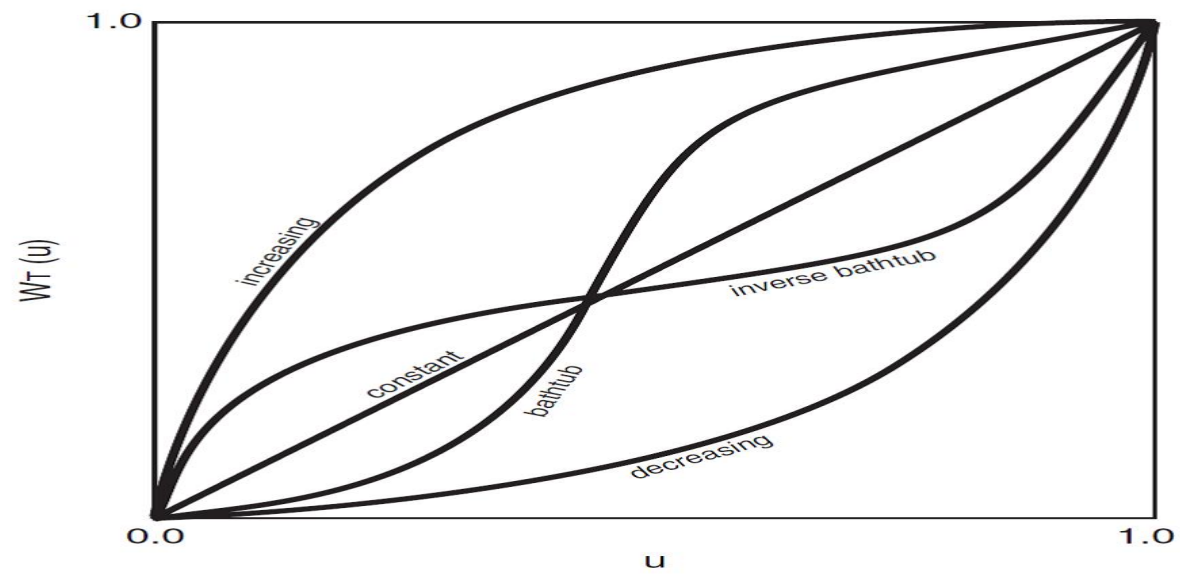

Figure 7: Theoretical aspects of TTT plots

\subsection{Real data examples with statistical analyses}

In order to check the competency we consider four data sets. Their descriptions and our statistical analyses are given below.

Data I: Generally it is observed that the brake pads of vehicles have a nominal lifetime, which is the number of miles or kilometers driven before the pads are reduced to a specified minimum thickness. To study the lifetime distribution, a manufacturer selected a random sample of 98 vehicles sold over the preceding 12 months to a specific group of dealers. Only cars that still had the initial pads were selected. For each car the brake pad lifetime $(x)$ could have then been observed by following the cars prospectively. Table 6 contains the life times of 98 vehicles given by [16].

Statistical analysis: The TTT plot of this data indicates an increasing failure rate pattern of the failure rate. Moreover, analysis of break pad data shows that the proposed model is the only suitable model from every aspects of data selection including the increasing hazard function. As we can see in Table 7, the proposed model have not only the minimum values of the test statistics and higher $p$ - value but also have least loss of information by showing least AIC, AICc, HQIC and CAIC. CDF plot also confirm this suitability behavior. 

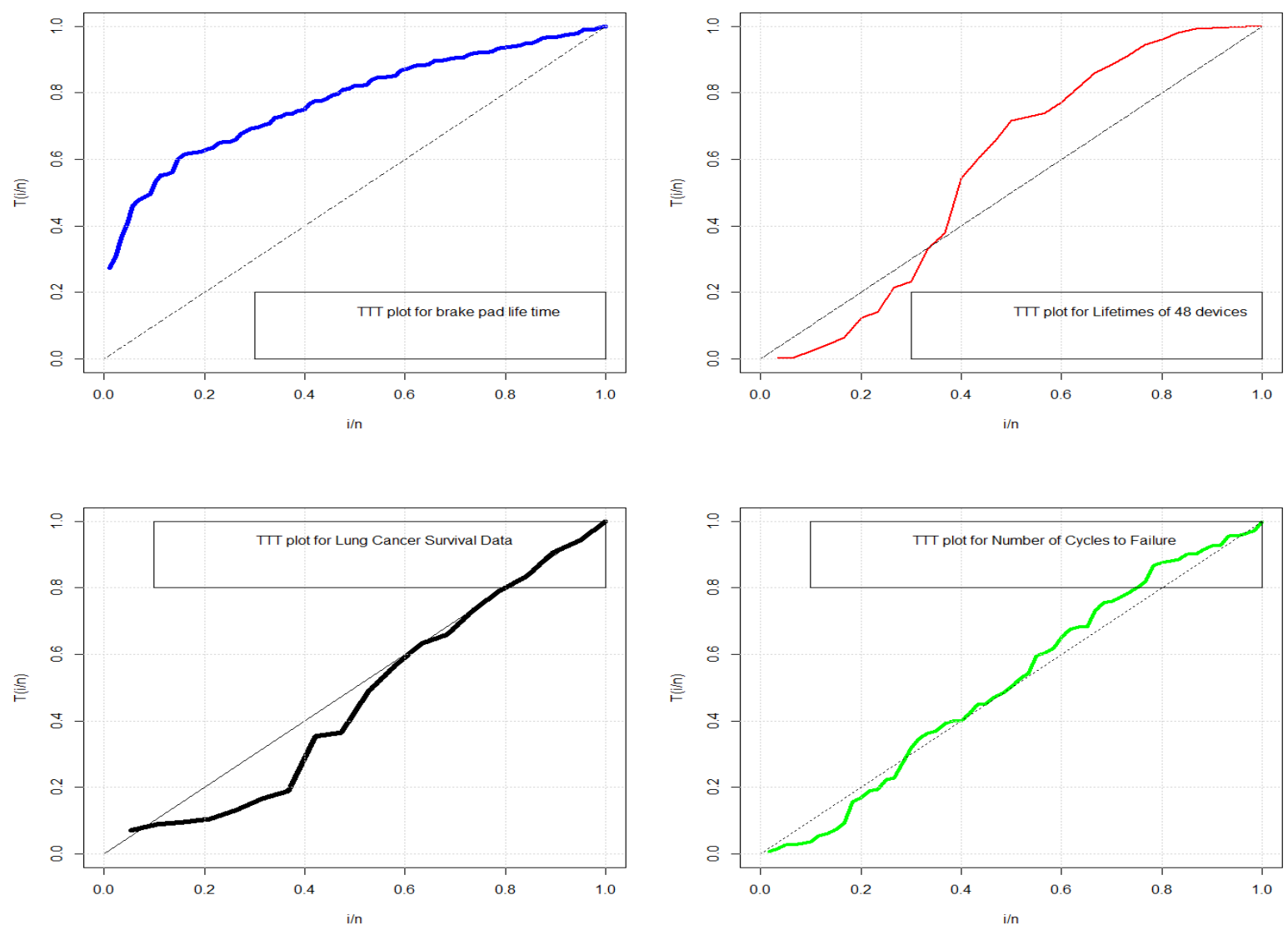

Figure 8: TTT plots of the selected data sets.

Data II: This data set contains the times to failure of 48 devices by [2] and reference therein. It can be found in Table 6 .

Statistical analysis: The data are known to have a bathtub-shaped failure rate as portrayed in TTT plot. In the analysis of the data the proposed model again shows a promising behavior with high $p$-value and lower Information criterion values which is portrayed in Table 8. Moreover CDF plot also shows that data is adequately modeled by the proposed distribution.

Data III: 21 advanced lung cancer patients, taken from a study discussed by [16], who were randomly assigned the chemotherapy treatments termed as "standard". Survival times t, measured from the start of treatment for each patient, are recorded in Table 6 .

Statistical analysis: Clearly the TTT plot of the current data set reveals decreasing hazard function pattern. Although the proposed distribution again shows minimum Log-Likelihood and higher $p$-value yet Sine Transformed shows AIC and HQIC value a little bit smaller than the proposed distribution which seems to be lager number of parameters plenty for the proposed distribution. But the proposed again achieved the adequate model fitting behavior by showing minimum AICc and CAIC which is portrayed in Table 9. Moreover CDF plot also shows the appropriateness of the proposed model.

Data IV: The data in Table 6 show the number of cycles to failure for a group of 60 electrical appliances in a life test. The failure times have been ordered for convenience. This data set is reported by $[16]$. 
a) CDFs of competing Distributions for brake pad life time

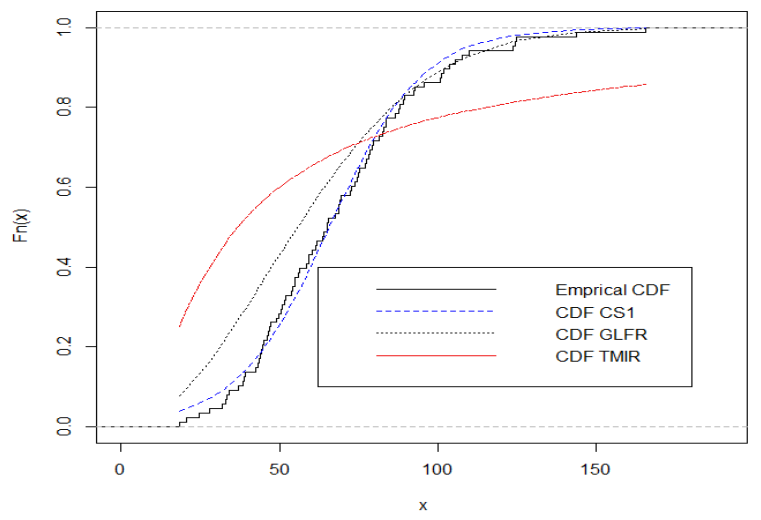

b) CDFs of competing Distributions for brake pad life time

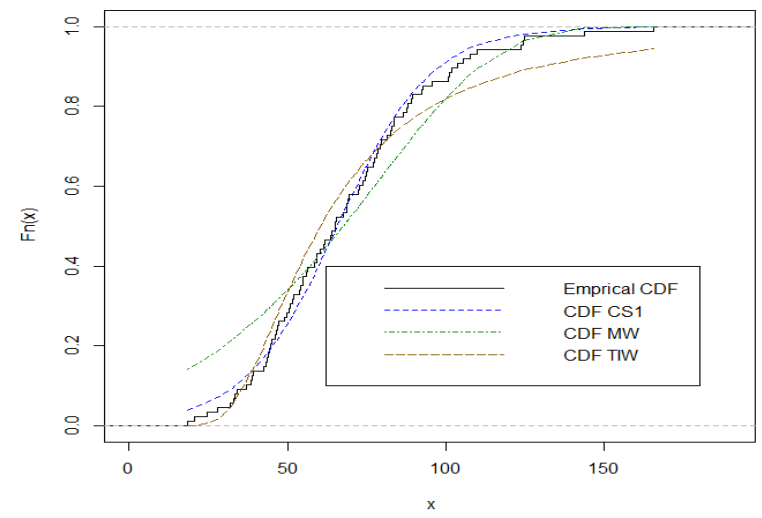

c) CDFs of competing Distributions for brake pad life time

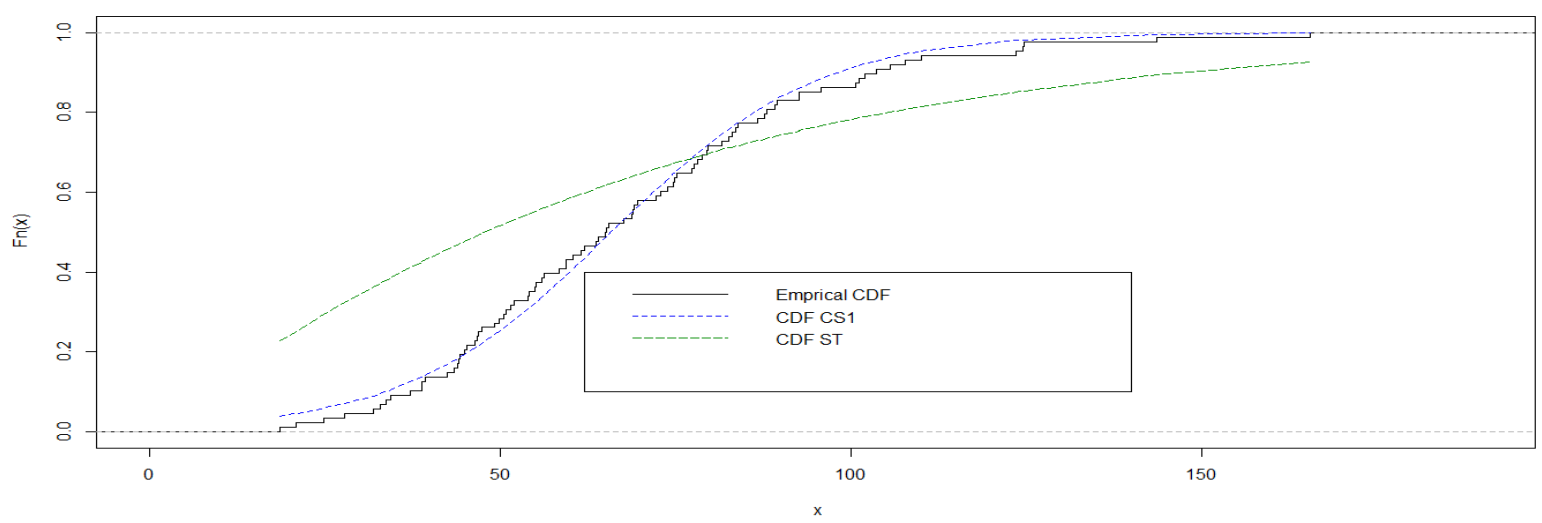

Statistical analysis: The TTT plot indicates that it follows the bathtub shape failure rate pattern. The analysis of the data is shown in Table 10 which clearly shows that proposed model is the most appropriate model for such data set, which is consolidated by CDF plot too.

\begin{tabular}{|c|c|}
\hline Data Set & Values \\
\hline I & $\begin{array}{c}38.7,49.2,42.4,73.8,46.7,44.1,61.9,39.3,49.8,46.3, \\
56.2,50.5,54.9,54.0,49.2,44.8,72.2,107.8,81.6,45.2, \\
124.6,64.0,83.0,143.6,43.4,69.6,74.8,32.9,51.5,31.8, \\
77.6,63.7,83.0,24.8,68.8,68.8,89.1,65.0,65.1,59.3, \\
53.9,79.4,47.4,61.4,72.8,54.0,37.2,44.2,50.8,65.5, \\
86.7,43.8,100.6,67.6,89.5,60.3,103.6,82.6,88.0,42.4, \\
68.9,95.7,78.1,83.6,18.6,92.6,42.4,34.3,105.6,20.8, \\
52.0,77.2,68.9,78.7,165.5,79.5,55.0,46.8,124.5,92.5, \\
110.0,101.2,59.4,27.8,33.6,69.0,75.2,58.4,105.6,56.2, \\
55.9,83.8,123.5,69.0,101.9,87.6,38.8,74.7\end{array}$ \\
\hline II & $\begin{array}{c}0.1,0.2,1,1,1,1,1,2,3,6 \\
7,11,12,18,18,18,18,18,21,32 \\
36,40,45,46,47,50,55,60,63,63 \\
67,67,67,67,72,75,79,82,82,83 \\
84,84,84,85,85,85,85,86\end{array}$ \\
\hline III & $\begin{array}{c}411,126,118,82,8,25,11,54,153,16 \\
56,21,287,10,8,12,177,12,200,250,100\end{array}$ \\
\hline IV & $\begin{array}{c}14,34,59,61,69,80,123,142,165,210, \\
\quad 381,464,479,556,574,839,917,969,991,1064, \\
1088,1091,1174,1270,1275,1355,1397,1477,1578,1649, \\
702,1893,1932,2001,2161,2292,2326,2337,2628,2785, \\
2811,2886,2993,3122,3248,3715,3790,3857,3912,4100, \\
410,4116,4315,4510,4584,5267,5299,5583,6065,9701 .\end{array}$ \\
\hline
\end{tabular}

Table 6: Data sets 
a) CDFs of competing Distributions for Lifetimes of 48 devices

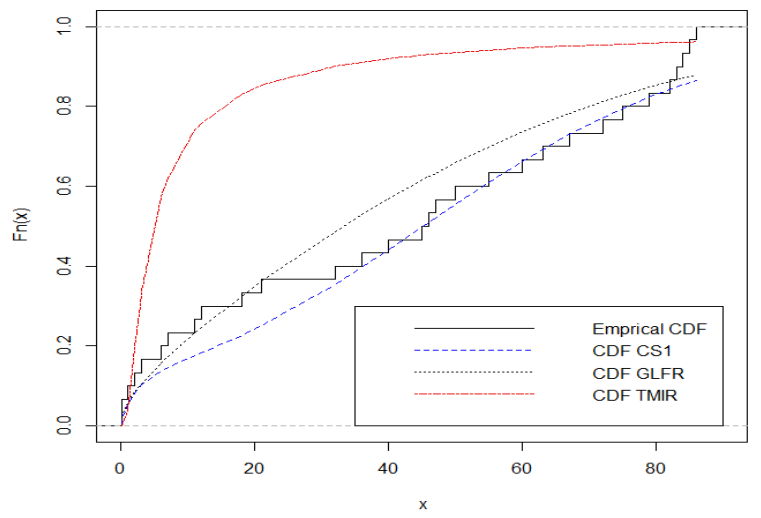

b) CDFs of competing Distributions for Lifetimes of $\mathbf{4 8}$ devices

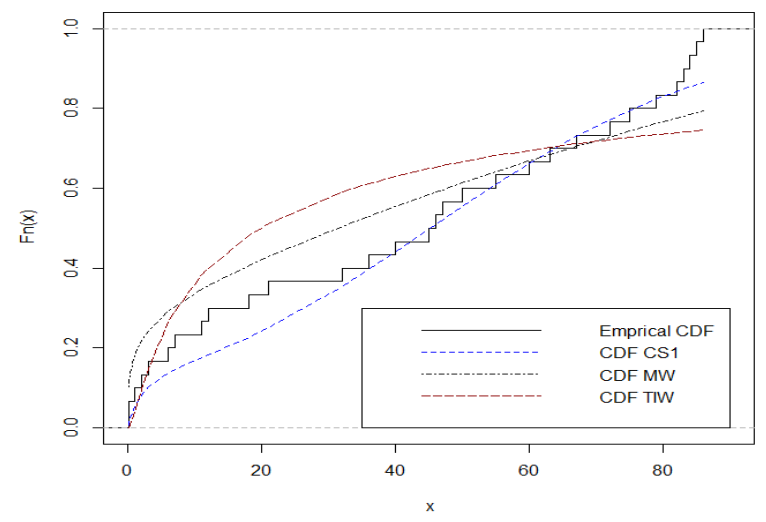

c) CDFs of competing Distributions for Lifetimes of 48 devices

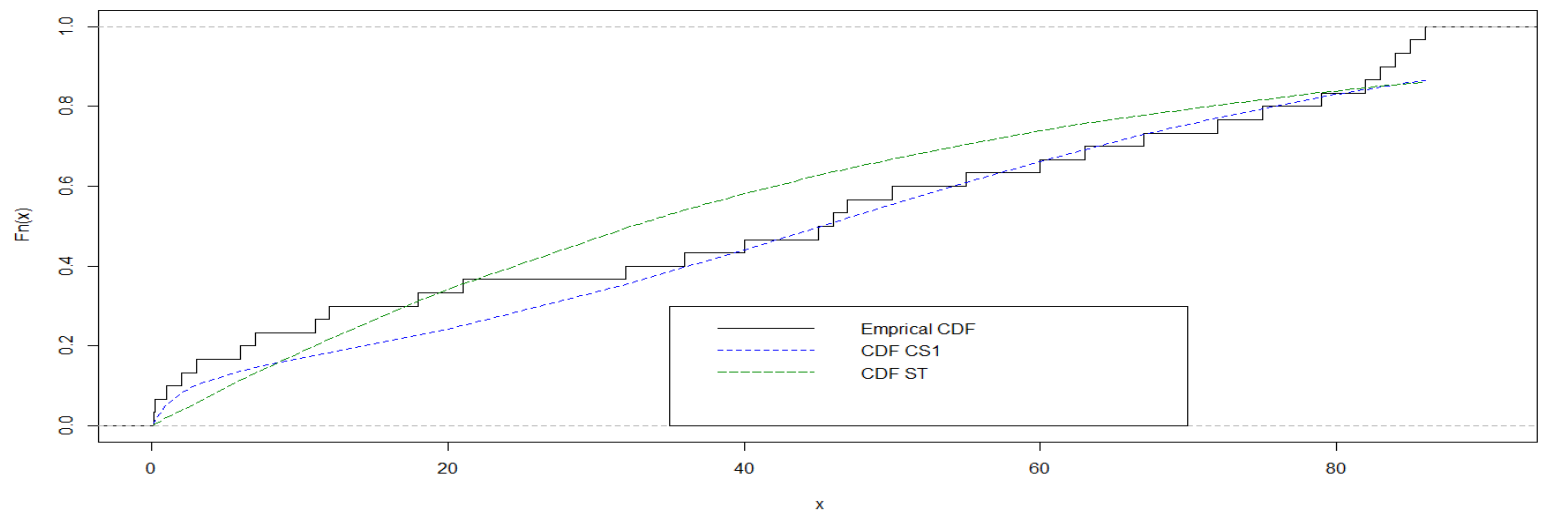

\begin{tabular}{ccccccccccccc}
\hline Distribution & $\hat{\alpha}$ & $\hat{\theta}$ & $\hat{\lambda}$ & $-\ell(\hat{\Theta})$ & KS & $p-$ value & $A_{0}^{*}$ & $W_{0}^{*}$ & AIC & AICc & HQIC & CAIC \\
\hline CS $1_{E}$ & 0.0408 & 2.2947 & 14.6839 & 459.4411 & 0.0554 & 0.763 & 0.5552 & 0.0565 & 924.8822 & 921.1271 & 928.0189 & 920.8822 \\
GLFRD & 0.0001 & 0.9733 & 0.0004 & 466.9339 & 0.1986 & 0.031 & 5.6182 & 1.0648 & 939.8678 & 936.1127 & 943.0045 & 935.8678 \\
MW & 0.2058 & 0.0518 & 0.0255 & 479.4219 & 0.1659 & 0.089 & 3.8553 & 0.5709 & 964.8439 & 961.0888 & 967.9806 & 960.8439 \\
TMIR & 25.4898 & 2.9973 & -0.0004 & 536.7692 & 0.4141 & 0.000 & 23.6871 & 4.9882 & 1079.538 & 1075.783 & 1082.675 & 1075.538 \\
TIW & 2.5011 & 41.7168 & -0.7856 & 465.9444 & 0.9465 & 0.000 & 68.2524 & 15.0603 & 937.8888 & 934.1337 & 941.0255 & 933.8888 \\
ST & - & 0.0085 & - & 506.0077 & 0.3231 & 0.000 & 14.5046 & 2.8601 & 1014.015 & 1012.056 & 1015.061 & 1012.015 \\
\hline
\end{tabular}

Table 7: Summary results for data set I

\begin{tabular}{cccccccccccccc}
\hline Distribution & $\hat{\alpha}$ & $\hat{\theta}$ & $\hat{\lambda}$ & $-\ell(\hat{\Theta})$ & KS & $p-$ value & $A_{0}^{*}$ & $W_{0}^{*}$ & AIC & AICc & HQIC & CAIC \\
\hline CS $1_{E}$ & 0.6240 & 3.0357 & 22.0911 & 218.6197 & 0.1351 & 0.578 & 0.7528 & 0.0816 & 443.2393 & 439.7393 & 445.3607 & 439.2393 \\
GLFRD & 0.0040 & 0.5231 & 0.0003 & 222.1862 & 0.1486 & 0.516 & 0.8760 & 0.1403 & 450.3724 & 446.8724 & 452.4937 & 446.3724 \\
MW & 0.2700 & 0.2002 & 0.0099 & 227.0751 & 0.2069 & 0.277 & 1.7361 & 0.2523 & 460.1501 & 456.6501 & 462.2715 & 456.1501 \\
TMIR & 1.7012 & 0.0010 & -0.9599 & 289.3668 & 0.5344 & 0.000 & 22.1076 & 3.1491 & 584.7336 & 581.2336 & 586.855 & 580.7336 \\
TIW & 0.3999 & 30.0910 & 0.9100 & 249.0317 & 0.8681 & 0.000 & 34.8866 & 6.3921 & 504.0634 & 500.5634 & 506.1848 & 500.0634 \\
ST & - & 0.01255 & - & 229.2952 & 0.16024 & 0.463 & 1.6232 & 0.1966 & 460.5904 & 458.6737 & 461.2975 & 458.5904 \\
\hline
\end{tabular}

Table 8: Summary results for data set II

\begin{tabular}{ccccccccccccccc}
\hline Distribution & $\hat{\alpha}$ & $\hat{\theta}$ & $\hat{\lambda}$ & $-\ell(\hat{\Theta})$ & KS & $p-$ value & $A_{0}^{*}$ & $W_{0}^{*}$ & AIC & AICc & HQIC & CAIC \\
\hline$C S 1_{E}$ & 26.3247 & 25.7904 & 94.3683 & 116.8890 & 0.1103 & 0.794 & 0.3030 & 0.0353 & 239.778 & 236.9209 & 240.4581 & 235.778 \\
GLFRD & 0.0081 & 0.8028 & $3.41 \times 10^{-6}$ & 117.8188 & 0.1109 & 0.792 & 0.4220 & 0.0589 & 241.6376 & 238.7805 & 242.3177 & 237.6376 \\
MW & 0.2099 & 0.2329 & 0.0037 & 123.7618 & 0.3101 & 0.161 & 1.5287 & 0.2511 & 253.5236 & 250.6665 & 254.2037 & 249.5236 \\
TMIR & 26.3251 & 14.7931 & 0.0004 & 118.5053 & 0.2502 & 0.304 & 1.5675 & 0.2849 & 243.0105 & 240.1534 & 243.6906 & 239.0105 \\
TIW & 0.8849 & 13.0655 & -0.9999 & 118.0363 & 0.9139 & 0.000 & 18.0276 & 3.7296 & 242.0726 & 239.2154 & 242.7526 & 242.7526 \\
ST & - & 0.0054 & - & 118.4882 & 0.1721 & 0.569 & 0.6439 & 0.0879 & 238.9764 & 237.1669 & 239.2031 & 236.9764 \\
\hline
\end{tabular}

Table 9: Summary results for data set III 

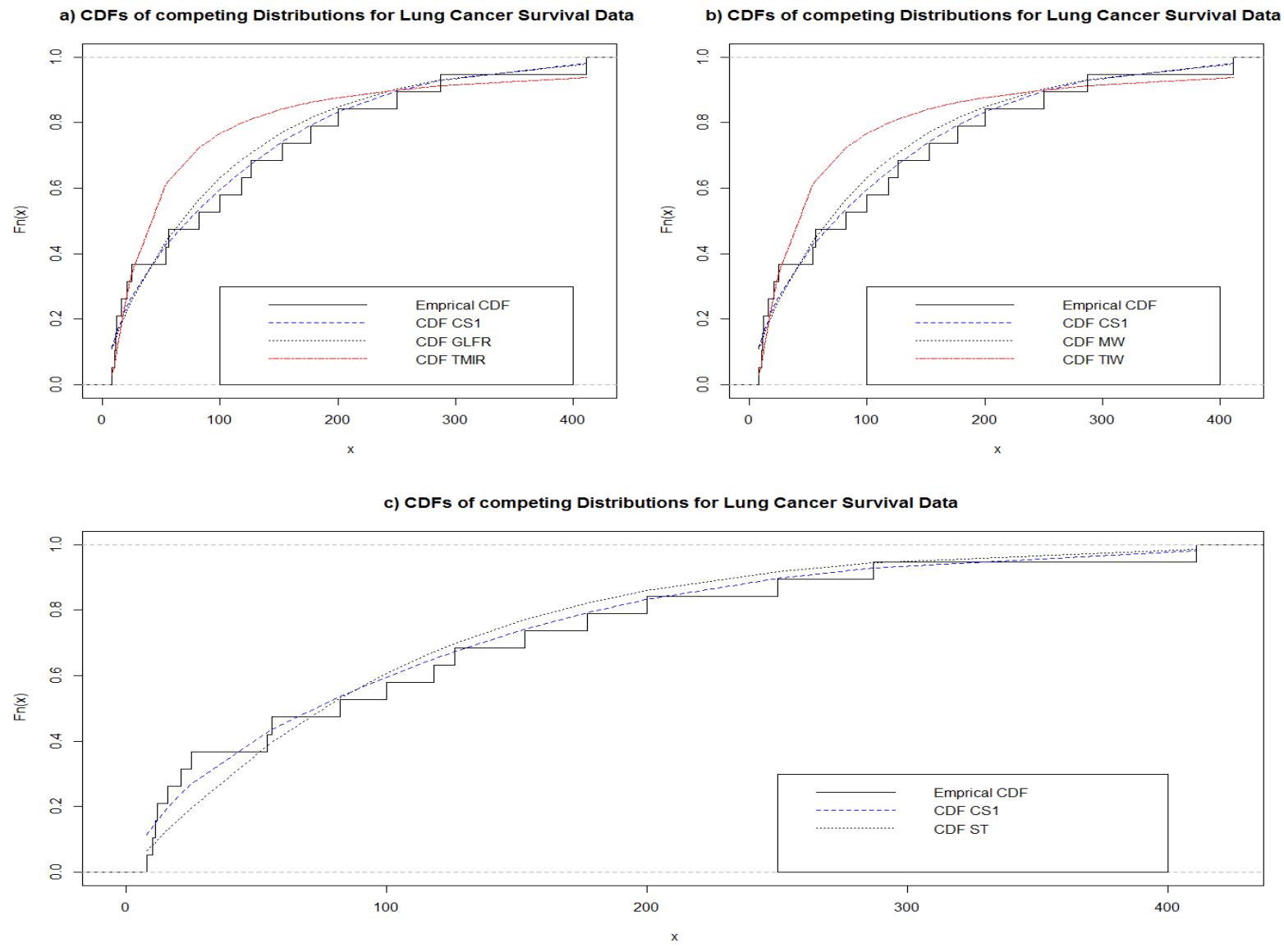

\begin{tabular}{cccccccccccccc}
\hline Distribution & $\hat{\alpha}$ & $\hat{\theta}$ & $\hat{\lambda}$ & $-\ell(\hat{\Theta})$ & KS & $p-$ value & $A_{0}^{*}$ & $W_{0}^{*}$ & AIC & AICc & HQIC & CAIC \\
\hline CS1E & 21.0763 & 42.4582 & 1379.0834 & 517.3469 & 0.0535 & 0.842 & 0.1791 & 0.02665 & 1040.694 & 1037.094 & 1043.151 & 1036.694 \\
GLFRD & 0.0006 & 1.4566 & $1.86 \times 10^{-8}$ & 523.9094 & 0.4077 & 0.000 & 48.1014 & 3.82674 & 1053.819 & 1050.219 & 1056.276 & 1049.819 \\
MW & 0.7658 & 0.0021 & 0.0001 & 517.9711 & 0.1189 & 0.428 & 2.0129 & 0.1455 & 1041.942 & 1038.342 & 1044.4 & 1037.942 \\
TMIR & 500.5075 & 0.0002 & 0.0011 & 580.4451 & 0.2802 & 0.009 & 10.7799 & 1.5494 & 1166.89 & 1163.29 & 1169.348 & 1162.89 \\
TIW & 0.5478 & 509.0170 & -0.1463 & 540.6114 & 0.8344 & 0.000 & 41.8053 & 9.1816 & 1087.223 & 1083.623 & 1089.68 & 1083.223 \\
ST & - & 0.0003 & - & 519.3744 & 0.0836 & 0.657 & 0.6689 & 0.0616 & 1040.749 & 1038.815 & 1043.568 & 1038.749 \\
\hline
\end{tabular}

Table 10: Summary results for data set IV

\section{Appendix}

Proof of Theorem 1. Let us now investigate the sufficient conditions for $G(x)$ to be a cdf.

- Since $(\alpha+\gamma) \sin \left(\frac{\pi}{2} F(x)\right)$ and $\alpha+\beta \cos \left(\frac{\pi}{2} F(x)\right)+\gamma \sin \left(\frac{\pi}{2} F(x)\right)+\theta \cos \left(\frac{\pi}{2} F(x)\right) \sin \left(\frac{\pi}{2} F(x)\right)$ are continuous functions with $\alpha+\beta \cos \left(\frac{\pi}{2} F(x)\right)+\gamma \sin \left(\frac{\pi}{2} F(x)\right)+\theta \cos \left(\frac{\pi}{2} F(x)\right) \sin \left(\frac{\pi}{2} F(x)\right) \neq 0$ owing to $\alpha \geq 0, \beta \geq 0, \gamma \geq 0, \theta \geq 0, \alpha+\gamma>0$ and $\alpha+\beta>0, G(x)$ is a continuous function of $x$.

- Let us prove that $G(x) \in[0,1]$. Since $\sin \left(\frac{\pi}{2} F(x)\right) \in[0,1], \cos \left(\frac{\pi}{2} F(x)\right) \in[0,1], \alpha \geq 0, \beta \geq 0$, $\gamma \geq 0, \theta \geq 0, \alpha+\gamma>0$ and $\alpha+\beta>0$, we have $G(x) \geq 0$. On the other hand, observe that $(\alpha+\gamma) \sin \left(\frac{\pi}{2} F(x)\right) \leq \alpha+\gamma \sin \left(\frac{\pi}{2} F(x)\right) \leq \alpha+\beta \cos \left(\frac{\pi}{2} F(x)\right)+\gamma \sin \left(\frac{\pi}{2} F(x)\right)+$ $\theta \cos \left(\frac{\pi}{2} F(x)\right) \sin \left(\frac{\pi}{2} F(x)\right)$. Hence $G(x) \leq 1$. 

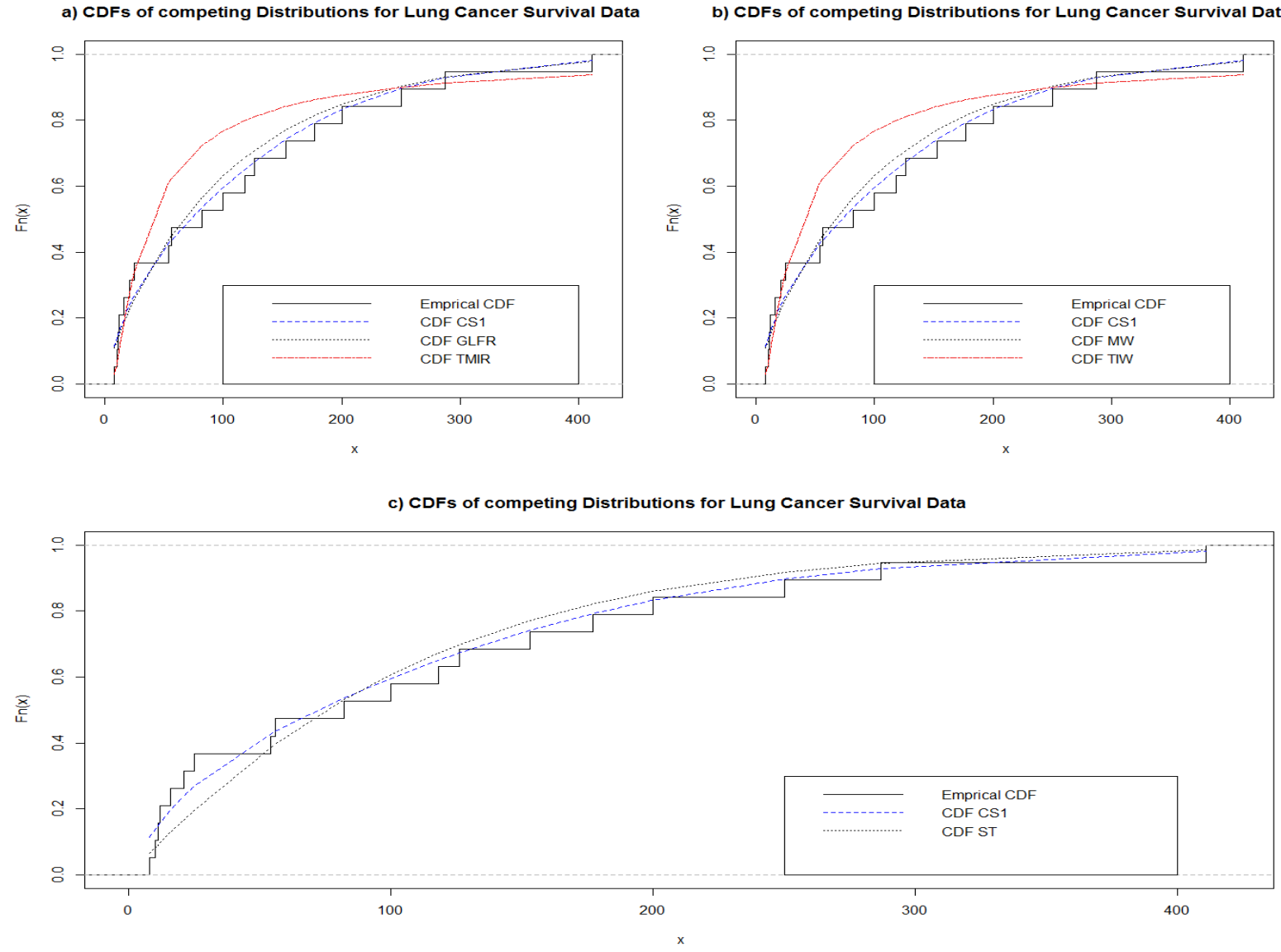

- Let us prove that $G^{\prime}(x) \geq 0$. We have $G^{\prime}(x)=\frac{u(x)}{v(x)}$ almost everywhere, where

$$
\begin{aligned}
u(x)= & (\alpha+\gamma) \frac{\pi}{2} f(x) \times \\
& \left(\cos \left(\frac{\pi}{2} F(x)\right)\left(\alpha+\beta \cos \left(\frac{\pi}{2} F(x)\right)+\gamma \sin \left(\frac{\pi}{2} F(x)\right)+\theta \cos \left(\frac{\pi}{2} F(x)\right) \sin \left(\frac{\pi}{2} F(x)\right)\right)\right. \\
- & \left.\sin \left(\frac{\pi}{2} F(x)\right)\left(-\beta \sin \left(\frac{\pi}{2} F(x)\right)+\gamma \cos \left(\frac{\pi}{2} F(x)\right)-\theta\left(\sin \left(\frac{\pi}{2} F(x)\right)\right)^{2}+\theta\left(\cos \left(\frac{\pi}{2} F(x)\right)\right)^{2}\right)\right) \\
= & (\alpha+\gamma) \frac{\pi}{2} f(x)\left(\beta+\alpha \cos \left(\frac{\pi}{2} F(x)\right)+\theta\left(\sin \left(\frac{\pi}{2} F(x)\right)\right)^{3}\right)
\end{aligned}
$$

and

$$
v(x)=\left(\alpha+\beta \cos \left(\frac{\pi}{2} F(x)\right)+\gamma \sin \left(\frac{\pi}{2} F(x)\right)+\theta \cos \left(\frac{\pi}{2} F(x)\right) \sin \left(\frac{\pi}{2} F(x)\right)\right)^{2}
$$

(some trigonometric formulas have been used to develop $u(x)$ ). Since $f(x) \geq 0, \sin \left(\frac{\pi}{2} F(x)\right) \in$ $[0,1], \cos \left(\frac{\pi}{2} F(x)\right) \in[0,1], \alpha \geq 0, \beta \geq 0, \gamma \geq 0, \theta \geq 0, \alpha+\gamma>0$ and $\alpha+\beta>0$, we have $u(x) \geq 0$ and $v(x)>0$, so $G^{\prime}(x) \geq 0$.

- Let us now investigate $\lim _{x \rightarrow-\infty} G(x)$ and $\lim _{x \rightarrow+\infty} G(x)$. Since $\lim _{x \rightarrow-\infty}(\alpha+\gamma) \sin \left(\frac{\pi}{2} F(x)\right)=(\alpha+$ $\gamma) \sin (0)=0$ and, using $\alpha+\beta>0, \lim _{x \rightarrow-\infty}\left(\alpha+\beta \cos \left(\frac{\pi}{2} F(x)\right)+\gamma \sin \left(\frac{\pi}{2} F(x)\right)+\theta \cos \left(\frac{\pi}{2} F(x)\right) \sin \left(\frac{\pi}{2} F(x)\right)\right)=$ $\alpha+\beta \cos (0)+\gamma \sin (0)+\theta \cos (0) \sin (0)=\alpha+\beta>0$, we have $\lim _{x \rightarrow-\infty} G(x)=0$. On the other hand, note that $\lim _{x \rightarrow+\infty}(\alpha+\gamma) \sin \left(\frac{\pi}{2} F(x)\right)=(\alpha+\gamma) \sin \left(\frac{\pi}{2}\right)=\alpha+\gamma$ and, since $\alpha+\gamma>0$, 


$$
\begin{aligned}
& \lim _{x \rightarrow+\infty}\left(\alpha+\beta \cos \left(\frac{\pi}{2} F(x)\right)+\gamma \sin \left(\frac{\pi}{2} F(x)\right)+\theta \cos \left(\frac{\pi}{2} F(x)\right) \sin \left(\frac{\pi}{2} F(x)\right)\right)=\alpha+\beta \cos \left(\frac{\pi}{2}\right)+\gamma \sin \left(\frac{\pi}{2}\right)+ \\
& \theta \cos \left(\frac{\pi}{2}\right) \sin \left(\frac{\pi}{2}\right)=\alpha+\gamma>0 . \text { Hence } \lim _{x \rightarrow+\infty} G(x)=1 .
\end{aligned}
$$

Proof of Proposition 1. We can write

$$
G(x)=\frac{\alpha+\gamma}{\nu} \sin \left(\frac{\pi}{2} F(x)\right) \frac{1}{1-H(x)},
$$

where $H(x)=\frac{\beta}{\nu}\left(1-\cos \left(\frac{\pi}{2} F(x)\right)\right)+\frac{\gamma}{\nu}\left(1-\sin \left(\frac{\pi}{2} F(x)\right)\right)+\frac{\theta}{\nu}\left(1-\cos \left(\frac{\pi}{2} F(x)\right) \sin \left(\frac{\pi}{2} F(x)\right)\right)$. Since $H(x) \in(0,1)$, the geometric series expansion gives

$$
G(x)=\frac{\alpha+\gamma}{\nu} \sin \left(\frac{\pi}{2} F(x)\right) \sum_{k=0}^{+\infty}[H(x)]^{k}
$$

Binomial series expansion gives

$$
\begin{aligned}
{[H(x)]^{k} } & =\sum_{\ell=0}^{k} \sum_{m=0}^{\ell}\left(\begin{array}{c}
k \\
\ell
\end{array}\right)\left(\begin{array}{c}
\ell \\
m
\end{array}\right)\left(\frac{\beta}{\nu}\right)^{k-\ell}\left(\frac{\theta}{\nu}\right)^{m}\left(\frac{\gamma}{\nu}\right)^{\ell-m}\left(1-\cos \left(\frac{\pi}{2} F(x)\right) \sin \left(\frac{\pi}{2} F(x)\right)\right)^{m} \\
& \times\left(1-\sin \left(\frac{\pi}{2} F(x)\right)\right)^{\ell-m}\left(1-\cos \left(\frac{\pi}{2} F(x)\right)\right)^{k-\ell} \cdot
\end{aligned}
$$

Again, with binomial series expansions, we get

$$
\begin{aligned}
{[H(x)]^{k} } & =\sum_{\ell=0}^{k} \sum_{m=0}^{\ell} \sum_{q=0}^{m} \sum_{r=0}^{\ell-m} \sum_{s=0}^{k-\ell}\left(\begin{array}{c}
k \\
\ell
\end{array}\right)\left(\begin{array}{c}
\ell \\
m
\end{array}\right)\left(\begin{array}{c}
m \\
q
\end{array}\right)\left(\begin{array}{c}
\ell-m \\
r
\end{array}\right)\left(\begin{array}{c}
k-\ell \\
s
\end{array}\right)\left(\frac{\beta}{\nu}\right)^{k-\ell}\left(\frac{\theta}{\nu}\right)^{m}\left(\frac{\gamma}{\nu}\right)^{\ell-m} \\
& \times(-1)^{q+r+s}\left(\cos \left(\frac{\pi}{2} F(x)\right)\right)^{q+s}\left(\sin \left(\frac{\pi}{2} F(x)\right)\right)^{q+r} \cdot
\end{aligned}
$$

Using the equation: $\cos ^{2}(x)=1-\sin ^{2}(x)$ and the generalized binomial series expansion, we get

$$
\begin{aligned}
{[H(x)]^{k} } & =\sum_{\ell=0}^{k} \sum_{m=0}^{\ell} \sum_{q=0}^{m} \sum_{r=0}^{\ell-m} \sum_{s=0}^{k-\ell} \sum_{t=0}^{+\infty}\left(\begin{array}{l}
k \\
\ell
\end{array}\right)\left(\begin{array}{c}
\ell \\
m
\end{array}\right)\left(\begin{array}{c}
m \\
q
\end{array}\right)\left(\begin{array}{c}
\ell-m \\
r
\end{array}\right)\left(\begin{array}{c}
k-\ell \\
s
\end{array}\right)\left(\begin{array}{c}
(q+s) / 2 \\
t
\end{array}\right)\left(\frac{\beta}{\nu}\right)^{k-\ell}\left(\frac{\theta}{\nu}\right)^{m}\left(\frac{\gamma}{\nu}\right)^{\ell-m} \\
& \times(-1)^{q+r+s+t}\left(\sin \left(\frac{\pi}{2} F(x)\right)\right)^{q+r+2 t} .
\end{aligned}
$$

Combining the equalities above, we end the proof of Proposition 1.

Proof of Proposition 2. Let $g(x)$ be the pdf given by (3). Then we have $\mathbb{E}(d(X))=\int_{-\infty}^{+\infty} d(x) g(x) d x$.

We can write

$$
g(x)=\frac{2 \pi \alpha}{(2 \alpha+\theta)^{2}}\left(\alpha \cos \left(\frac{\pi}{2} F(x)\right)+\theta\left(\sin \left(\frac{\pi}{2} F(x)\right)\right)^{3}\right) f(x) \frac{1}{(1-H(x))^{2}},
$$

where $H(x)=\frac{\theta}{2 \alpha+\theta}(1-\sin (\pi F(x)))$. Since $H(x) \in(0,1)$, the geometric series expansion gives

$$
g(x)=\frac{2 \pi \alpha}{(2 \alpha+\theta)^{2}}\left(\alpha \cos \left(\frac{\pi}{2} F(x)\right)+\theta\left(\sin \left(\frac{\pi}{2} F(x)\right)\right)^{3}\right) f(x) \sum_{k=0}^{+\infty}(k+1)[H(x)]^{k} .
$$

On the other hand, the binomial series expansion gives

$$
[H(x)]^{k}=\frac{\theta^{k}}{(2 \alpha+\theta)^{k}} \sum_{\ell=0}^{k}\left(\begin{array}{l}
k \\
\ell
\end{array}\right)(-1)^{\ell}(\sin (\pi F(x)))^{\ell} .
$$


By putting these equalities together, we end the proof of Proposition 2.

Proof of Proposition 3. Let $g(x)$ be the pdf given by (8). Then we have $\mathbb{E}(d(X))=\int_{-\infty}^{+\infty} d(x) g(x) d x$. We can write

$$
g(x)=\frac{\pi \gamma \beta}{2(\alpha+\beta)^{2}} f(x) \frac{1}{(1-H(x))^{2}},
$$

where $H(x)=\frac{\beta}{\alpha+\beta}\left(1-\cos \left(\frac{\pi}{2} F(x)\right)\right)+\frac{\alpha}{\alpha+\beta}\left(1-\sin \left(\frac{\pi}{2} F(x)\right)\right)$. Since $H(x) \in(0,1)$, the geometric series expansion gives

$$
g(x)=\frac{\pi \gamma \beta}{2(\alpha+\beta)^{2}} f(x) \sum_{k=0}^{+\infty}(k+1)[H(x)]^{k} .
$$

On the other hand, the binomial series expansion gives

$$
\begin{aligned}
{[H(x)]^{k} } & =\sum_{\ell=0}^{k}\left(\begin{array}{l}
k \\
\ell
\end{array}\right) \frac{\beta^{\ell}}{(\alpha+\beta)^{\ell}}\left(1-\cos \left(\frac{\pi}{2} F(x)\right)\right)^{\ell} \frac{\alpha^{k-\ell}}{(\alpha+\beta)^{k-\ell}}\left(1-\sin \left(\frac{\pi}{2} F(x)\right)\right)^{k-\ell} \\
& =\sum_{\ell=0}^{k} \sum_{m=0}^{\ell} \sum_{s=0}^{k-\ell}\left(\begin{array}{c}
k \\
\ell
\end{array}\right)\left(\begin{array}{c}
\ell \\
m
\end{array}\right)\left(\begin{array}{c}
k-\ell \\
s
\end{array}\right) \frac{\beta^{\ell}}{(\alpha+\beta)^{\ell}}(-1)^{m+s} \frac{\alpha^{k-\ell}}{(\alpha+\beta)^{k-\ell}}\left(\cos \left(\frac{\pi}{2} F(x)\right)\right)^{m}\left(\sin \left(\frac{\pi}{2} F(x)\right)\right)^{s} .
\end{aligned}
$$

Using the equation: $\cos ^{2}(x)=1-\sin ^{2}(x)$ and the generalized binomial series expansion, we get

$$
[H(x)]^{k}=\sum_{\ell=0}^{k} \sum_{m=0}^{\ell} \sum_{s=0}^{k-\ell} \sum_{t=0}^{+\infty}\left(\begin{array}{c}
k \\
\ell
\end{array}\right)\left(\begin{array}{c}
\ell \\
m
\end{array}\right)\left(\begin{array}{c}
k-\ell \\
s
\end{array}\right)\left(\begin{array}{c}
m / 2 \\
t
\end{array}\right) \frac{\beta^{\ell}}{(\alpha+\beta)^{\ell}}(-1)^{m+s+t} \frac{\alpha^{k-\ell}}{(\alpha+\beta)^{k-\ell}}\left(\sin \left(\frac{\pi}{2} F(x)\right)\right)^{s+2 t} .
$$

By putting these equalities together, we end the proof of Proposition 3.

\section{References}

[1] Al-Faris, R. Q. and Khan, S. (2008). Sine Square Distribution: A New Statistical Model Based on the Sine Function. Journal of Applied Probability 6 Statistics, 3(1),163-173.

[2] Almalki, S. J. (2014). Statistical Analysis of Lifetime Data Using New Modified Weibull Distributions (Unpublished doctoral dissertation). The University of Manchester, UK.

[3] Evans, M., Hastings, N. and Peacock, B. (2000). Statistical Distributions, 3rd ed. New York, Wiley.

[4] Fisher, N. I. (1993). Statistical Analysis of Directional Data, Cambridge University Press,UK.

[5] Gupta, R. D. and Kundu, D. (1999). Generalized Exponential Distributions. Australian and New Zealand Journal of Statistics, 41(2), 173-188.

[6] Hamedani, G. G. (2016). On characterizations and infinite divisibility of recently introduced distribution. Studia Scientiarum Mathematicarum Hungarica, 53, 4, 467-511.

[7] Hamedani, G. G. and Safavimanesh, F. (2017). Characterizations and Infinite Divisibility of Certain 2016 Univariate Continuous Distributions. International Mathematical Forum, 12, 5, 195-228.

[8] Hamedani, G. G. (2017). Characterizations and Infinite Divisibility of Certain 2016-2017 Univariate Continuous Distributions II. International Mathematical Forum, 12, 12, 565 - 609.

[9] Kent, J. T. and Tyler, D. E. (1988). Maximum likelihood estimation for the wrapped Cauchy distribution. J. Appl. Statist., 15 247-254. 
[10] Khan, M. S. and King, R. (2015). Transmuted Modified Inverse Rayleigh Distribution. Austrian Journal of Statistics, 44, 17-29.

[11] Khan, M. S., King, R. and Hudson, I. L. (2014). Characterizations of the transmuted inverse Weibull distribution. ANZIAM Journal, 55, 197-217.

[12] Kotz, S. and Johanson, N. L. (1982). Circular normal distribution. Encyclopedia of Sta- tistical Sciences, 1,479 .

[13] Kumar, D., Singh, U. and Singh, S. K. (2015). A Method of Proposing New Distribution and its Application to Bladder Cancer Patients Data. J. Stat. Appl. Probability Letter, 2 (3), 235- 245.

[14] Kumar, D., Singh, U. and Singh, S. K. (2015). A New Distribution Using Sine Function- Its Application to Bladder Cancer Patients Data. J. Stat. Appl. Pro., 4 (3), 417- 427.

[15] Lai, C. D., Xie, M. and Murthy, D. N. P. (2003). A modified Weibull distribution, IEEE Transactions on Reliability, 52, 33-37.

[16] Lawless, J. F. (2003). Statistical Models and Methods for Lifetime data $2^{\text {nd }}$ Edition. John Wiley \& Sons, Inc., Floboken, New Jersey.

[17] Nadarajah, S. and Kotz, S. (2006). Beta Trigonometric Distribution. Portuguese Economic Journal, 5(3),207-224.

[18] Sarhan, A.M. and Kundu, D. (2009). Generalized Linear Failure Rate Distribution. Communications in Statistics - Theory and Methods, 38(5), 642-660.

[19] Shaw, W. T. and Buckley, I. R. C. (2009). The alchemy of probability distributions: beyond Gram-Charlier expansions, and a skew-kurtotic-normal distribution from a rank transmutation map, arXiv preprint arXiv:0901.0434.

[20] Tahir, M.H. and Cordeiro, G.M. (2016). Compounding of distributions: a survey and new generalized classes. Journal of Statistical Distributions and Applications, 3 (1), 1-35. 